\title{
ON INDECOMPOSABLE VERTEX ALGEBRAS ASSOCIATED WITH VERTEX ALGEBROIDS
}

\author{
PHICHET JITJANKARN, AND GAYWALEE YAMSKULNA
}

\begin{abstract}
Let $A$ be a finite dimensional unital commutative associative algebra and let $B$ be a finite dimensional vertex $A$-algebroid such that its Levi factor is isomorphic to $s l_{2}$. Under suitable conditions, we construct an indecomposable non-simple $\mathbb{N}$-graded vertex algebra $\overline{V_{B}}$ from the $\mathbb{N}$-graded vertex algebra $V_{B}$ associated with the vertex $A$-algebroid $B$. We show that this indecomposable non-simple $\mathbb{N}$-graded vertex algebra $\overline{V_{B}}$ is $C_{2}$-cofinite and has only two irreducible modules.
\end{abstract}

\section{INTRODUCTION}

It is well known that the $C_{2}$-cofiniteness property of vertex (operator) algebras plays an important role in the study of representation theory of vertex (operator) algebras (e.g. [A2], [ABD], $[\mathrm{Bu}]$, DoLiM1, GN], Mi1-[Mi3], [Z]). Over the years, rational $C_{2}$-cofinite vertex algebras have been studied intensively (e.g. [Bo1-[Bo2], [Do], [DoL], DoLiM1], DoM1], [FrLMe1, [FZ]) . However, the literature devoted to the study of irrational $C_{2}$-cofinite vertex algebras is sparse. In fact, there are very few known examples of families of irrational $C_{2}$-cofinite vertex (super)algebras (e.g. [A1], AdM1- AdM4, [CF], [FFHST], [FGST1-[FGST3]). Therefore, it is crucial to study these known examples and to seek for new models.

The aim of this paper is to construct indecomposable non-simple vertex algebras that satisfy the $C_{2}$-condition from $\mathbb{N}$-graded vertex algebras associated with vertex algebroids. For a $\mathbb{N}$ graded vertex algebra $V=\oplus_{n=0}^{\infty} V_{(n)}$ such that $\operatorname{dim} V_{(0)} \geq 2$, it is known widely that $V_{(0)}$ is a unital commutative associative algebra and $V_{(1)}$ is a vertex $V_{(0)}$-algebroid. In [GMS], among other important things, Gorbounov, Malikov and Schechtman constructed a $\mathbb{N}$-graded vertex algebra $V=\oplus_{n=0}^{\infty} V_{(n)}$ from any vertex $A$-algebroid, such that $V_{(0)}=A$ and the vertex $A$-algebroid $V_{(1)}$ is isomorphic to the given vertex $A$-algebroid. The classification of graded simple non-twisted and twisted modules for the vertex algebras associated with vertex algebroids had been studied in [LiY1]-[LiY2] by Li and the second author of this paper.

In terms of general theory of $\mathbb{N}$-graded vertex algebras, Dong and Mason showed that a $\mathbb{N}$ graded vertex operator algebra $V$ is local if and only if $V_{(0)}$ is a local algebra. Moreover, indecomposibility of $V$ is equivalent to $V_{(0)}$ being a local algebra DoM2. Note that in order to prove this statement one needs to have a Virasoro element. In [JY], we explored criteria for $\mathbb{N}$-graded vertex algebras $V=\oplus_{n=0}^{\infty} V_{(n)}$ such that $\operatorname{dim} V_{(0)} \geq 2$ to be indecomposable non-simple vertex algebras and studied influences of semisimple Leibniz algebras on

Key words and phrases. $C_{2}$-cofinite, indecomposable, irrational vertex algebras. 
the algebraic structure of this type of vertex algebras. Precisely, we provided tools to characterize indecomposable non-simple $\mathbb{N}$-graded vertex algebras. Also, we examined the algebraic structure of $\mathbb{N}$-graded vertex algebras $V=\oplus_{n=0}^{\infty} V_{(n)}$ that is generated by $V_{(0)}$ and $V_{(1)}$ such that $\operatorname{dim} V_{(0)} \geq 2$ and $V_{(1)}$ is a (semi)simple Leibniz algebra that has $s l_{2}$ as its Levi factor. We showed that under suitable conditions this type of vertex algebra is indecomposable non-simple.

In this paper, we continue our investigation on $\mathbb{N}$-graded indecomposable non-simple vertex algebras. First, we apply results in JY] to show that for a given finite dimensional vertex $A$-algebroid $B$ such that $B$ is a (semi)simple Leibniz algebra that has $s l_{2}$ as its Levi factor, the $\mathbb{N}$-graded vertex algebra associated with the vertex $A$-algebroid $B$ is indecomposable and non-simple. Moreover, we establish the following results.

Theorem 1. Let $A$ be a finite-dimensional commutative associative algebra with the identity $\mathfrak{e}$ such that $\operatorname{dim} A \geq 2$. Let $B$ be a finite-dimensional vertex $A$-algebroid such that $A$ is not a trivial $B$-module and Leib $(B) \neq\{0\}$. Let $S$ be the Levi factor of the Leibniz algebra $B$ such that $S=\operatorname{Span}\{e, f, h\}, e_{0} f=h, h_{0} e=2 e, h_{0} f=-2 f$, and $e_{1} f=k \mathfrak{e}$. Here, $k \in \mathbb{C} \backslash\{0\}$. Assume that one of the following statements hold.

(I) $B$ is simple Leibniz algebra;

(II) $B$ is a semisimple Leibniz algebra and $\operatorname{Ker}(\partial)=\left\{a \in A \mid b_{0} a=0\right.$ for all $\left.b \in B\right\}$.

We then have the following results:

(i) the $\mathbb{N}$-graded vertex algebra $V_{B}\left(=\oplus_{n=0}^{\infty}\left(V_{B}\right)_{(n)}\right)$ associated with the vertex $A$-algebroid $B$ is indecomposable non-simple.

(ii) The set of representatives of equivalence classes of finite-dimensional simple sl $_{2}$-modules is equivalent to the set of representatives of equivalence classes of $\mathbb{N}$-graded simple $V_{B}$-modules $N=\oplus_{n=0}^{\infty} N_{(n)}$ such that $\operatorname{dim} N_{(0)}<\infty$.

Next, we show that a certain quotient space of $V_{B}$, constructed in Theorem 1 , is an indecomposable non-simple vertex algebra that satisfies the $C_{2}$-condition and has only two irreducible modules. Precisely, we establish the following results.

Theorem 2. Let $A$ be a finite-dimensional commutative associative algebra with the identity $\mathfrak{e}$ such that $\operatorname{dim} A \geq 2$. Let $B$ be a finite-dimensional vertex $A$-algebroid that satisfies the given conditions in Theorem 1. Let $(e(-1) e)$ be an ideal of $V_{B}$ that is generated by $e(-1) e$. Then

(i) $(e(-1) e) \cap A=\{0\},(e(-1) e) \cap B=\{0\}$. Moreover, the $\mathbb{N}$-graded vertex algebra $V_{B} /(e(-1) e)$ is indecomposable non-simple.

(ii) The vertex algebra $V_{B} /(e(-1) e)$ satisfies the $C_{2}$-condition.

(iii) Let $L=\mathbb{Z} \alpha$ be a rank one positive definite even lattice equipped with a $\mathbb{Q}$-valued $\mathbb{Z}$ bilinear form $(\cdot, \cdot)$ such that $(\alpha, \alpha)=2$. Then $V_{L}$ and $V_{L+\frac{1}{2} \alpha}$ are the only two irreducible $V_{B} /(e(-1) e)$-modules. 
This paper is organized as follows: in Section 2, we first review properties of Leibniz algebras, 1-truncated conformal algebras, and vertex algebroids. We discuss about vertex algebroids associated with (semi) simple Leibniz algebras that have $s l_{2}$ as their Levi factor. Also, we give necessary background on vertex algebras and recall construction of vertex algebras associated with vertex algebroids, and their graded simple modules. In Section 3, we give the proof of Theorem 1, and Theorem 2, We include some appendices in Section 4 containing background on a vertex operator algebra associated with a certain type of rank one positive definite even lattices, and vertex operator algebras associated with highest weight representations of affine Lie algebras.

\section{Preliminaries}

\subsection{Leibniz Algebras.}

Definition 3. ([DMS], [FM])

(i) A left Leibniz algebra $\mathfrak{L}$ is a $\mathbb{C}$-vector space equipped with a bilinear map $[]:, \mathfrak{L} \times \mathfrak{L} \rightarrow \mathfrak{L}$ satisfying the Leibniz identity $[a,[b, c]]=[[a, b], c]+[b,[a, c]]$ for all $a, b, c \in \mathfrak{L}$.

(ii) Let $\mathfrak{L}$ be a left Leibniz algebra over $\mathbb{C}$. Let $I$ be a subspace of $\mathfrak{L}$. $I$ is a left (respectively, right) ideal of $\mathfrak{L}$ if $[\mathfrak{L}, I] \subseteq I$ (respectively, $[I, \mathfrak{L}] \subseteq I$ ). I is an ideal of $\mathfrak{L}$ if it is both a left and a right ideal.

Example 4. We define $\operatorname{Leib}(\mathfrak{L})=\operatorname{Span}\{[u, u] \mid u \in \mathfrak{L}\}=\operatorname{Span}\{[u, v]+[v, u] \mid u, v \in \mathfrak{L}\}$. $\operatorname{Leib}(\mathfrak{L})$ is an ideal of $\mathfrak{L}$. Moreover, for $v, w \in \operatorname{Leib}(\mathfrak{L}),[v, w]=0$.

Definition 5. DMS Let $(\mathfrak{L},[]$,$) be a left Leibniz algebra. The series of ideals$

$$
\ldots \subseteq \mathfrak{L}^{(2)} \subseteq \mathfrak{L}^{(1)} \subseteq \mathfrak{L}
$$

where $\mathfrak{L}^{(1)}=[\mathfrak{L}, \mathfrak{L}], \mathfrak{L}^{(i+1)}=\left[\mathfrak{L}^{(i)}, \mathfrak{L}^{(i)}\right]$ is called the derived series of $\mathfrak{L}$. A left Leibniz algebra $\mathfrak{L}$ is solvable if $\mathfrak{L}^{(m)}=0$ for some integer $m \geq 0$. As in the case of Lie algebras, any left Leibniz algebra $\mathfrak{L}$ contains a unique maximal solvable ideal $\operatorname{rad}(\mathfrak{L})$ called the the radical of $\mathfrak{L}$ which contains all solvable ideals.

Example 6. Leib( $\mathfrak{L})$ is a solvable ideal.

Definition 7. DMS

(i) A left Leibniz algebra $\mathfrak{L}$ is simple if $[\mathfrak{L}, \mathfrak{L}] \neq \operatorname{Leib}(\mathfrak{L})$, and $\{0\}, \operatorname{Leib}(\mathfrak{L}), \mathfrak{L}$ are the only ideals of $\mathfrak{L}$.

(ii) A left Leibniz algebra $\mathfrak{L}$ is said to be semisimple if $\operatorname{rad}(\mathfrak{L})=\operatorname{Leib}(\mathfrak{L})$.

Proposition 8. ([Ba], $[\mathrm{DMS}]$ ) Let $\mathfrak{L}$ be a left Leibniz algebra.

(i) There exists a subalgebra $S$ which is a semisimple Lie algebra of $\mathfrak{L}$ such that $\mathfrak{L}=S \dot{+}$ rad( $(\mathfrak{L})$. As in the case of a Lie algebra, we call $S$ a Levi subalgebra or a Levi factor of $\mathfrak{L}$.

(ii) If $\mathfrak{L}$ is a semisimple Leibniz algebra then $\mathfrak{L}=\left(S_{1} \oplus S_{2} \oplus \ldots \oplus S_{k}\right) \dot{+}$ Leib( $\left.\mathfrak{L}\right)$, where $S_{j}$ is a simple Lie algebra for all $1 \leq j \leq k$. Moreover, $[\mathfrak{L}, \mathfrak{L}]=\mathfrak{L}$. 
(iii) If $\mathfrak{L}$ is a simple Leibniz algebra, then there exists a simple Lie algebra $S$ such that Leib( $\mathfrak{L})$ is an irreducible module over $S$ and $\mathfrak{L}=S \dot{+} \operatorname{Leib}(\mathfrak{L})$.

Definition 9. Let $\mathfrak{L}$ be a left Leibniz algebra. A left $\mathfrak{L}$-module is a vector space $M$ equipped with a $\mathbb{C}$-bilinear map $\mathfrak{L} \times M \rightarrow M ;(u, m) \mapsto u \cdot m$ such that $([u, v]) \cdot m=u \cdot(v \cdot m)-v \cdot(u \cdot m)$ for all $u, v \in \mathfrak{L}, m \in M$.

The usual definitions of the notions of submodule, irreducibility, complete reducibility, homomorphism, isomorphism, etc., hold for left Leibniz modules.

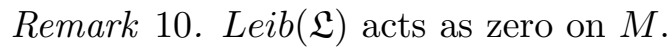

\subsection{1-Truncated Conformal Algebras and Vertex Algebroids.}

Definition 11. GMS A 1-truncated conformal algebra is a graded vector space $C=C_{0} \oplus C_{1}$ equipped with a linear map $\partial: C_{0} \rightarrow C_{1}$ and bilinear operations $(u, v) \mapsto u_{i} v$ for $i=0,1$ of degree $-i-1$ on $C=C_{0} \oplus C_{1}$ such that the following axioms hold:

(Derivation) for $a \in C_{0}, u \in C_{1}$,

$$
(\partial a)_{0}=0, \quad(\partial a)_{1}=-a_{0}, \quad \partial\left(u_{0} a\right)=u_{0} \partial a ;
$$

(Commutativity) for $a \in C_{0}, u, v \in C_{1}$,

$$
u_{0} a=-a_{0} u, \quad u_{0} v=-v_{0} u+\partial\left(u_{1} v\right), \quad u_{1} v=v_{1} u
$$

(Associativity) for $\alpha, \beta, \gamma \in C_{0} \oplus C_{1}$,

$$
\alpha_{0} \beta_{i} \gamma=\beta_{i} \alpha_{0} \gamma+\left(\alpha_{0} \beta\right)_{i} \gamma .
$$

Definition 12. ([Br1, [Br2], GMS] $)$ Let $(A, *)$ be a unital commutative associative algebra over $\mathbb{C}$ with the identity 1 . A vertex $A$-algebroid is a $\mathbb{C}$-vector space $\Gamma$ equipped with

(1) a $\mathbb{C}$-bilinear map $A \times \Gamma \rightarrow \Gamma, \quad(a, v) \mapsto a \cdot v$ such that $1 \cdot v=v$ (i.e. a nonassociative unital $A$-module),

(2) a structure of a Leibniz $\mathbb{C}$-algebra $[]:, \Gamma \times \Gamma \rightarrow \Gamma$,

(3) a homomorphism of Leibniz $\mathbb{C}$-algebra $\pi: \Gamma \rightarrow \operatorname{Der}(A)$,

(4) a symmetric $\mathbb{C}$-bilinear pairing $\langle\rangle:, \Gamma \otimes_{\mathbb{C}} \Gamma \rightarrow A$,

(5) a $\mathbb{C}$-linear map $\partial: A \rightarrow \Gamma$ such that $\pi \circ \partial=0$ which satisfying the following conditions:

$$
\begin{aligned}
& a \cdot\left(a^{\prime} \cdot v\right)-\left(a * a^{\prime}\right) \cdot v=\pi(v)(a) \cdot \partial\left(a^{\prime}\right)+\pi(v)\left(a^{\prime}\right) \cdot \partial(a), \\
& {[u, a \cdot v]=\pi(u)(a) \cdot v+a \cdot[u, v],} \\
& {[u, v]+[v, u]=\partial(\langle u, v\rangle),} \\
& \pi(a \cdot v)=a \pi(v), \\
& \langle a \cdot u, v\rangle=a *\langle u, v\rangle-\pi(u)(\pi(v)(a)), \\
& \pi(v)\left(\left\langle v_{1}, v_{2}\right\rangle\right)=\left\langle\left[v, v_{1}\right], v_{2}\right\rangle+\left\langle v_{1},\left[v, v_{2}\right]\right\rangle, \\
& \partial\left(a * a^{\prime}\right)=a \cdot \partial\left(a^{\prime}\right)+a^{\prime} \cdot \partial(a), \\
& {[v, \partial(a)]=\partial(\pi(v)(a)),} \\
& \langle v, \partial(a)\rangle=\pi(v)(a)
\end{aligned}
$$

for $a, a^{\prime} \in A, u, v, v_{1}, v_{2} \in \Gamma$. 
Proposition 13. LiY1 Let $(A, *)$ be a unital commutative associative algebra and let $B$ be a module for $A$ as a nonassociative algebra. Then a vertex A-algebroid structure on $B$ exactly amounts to a 1-truncated conformal algebra structure on $C=A \oplus B$ with

$$
\begin{aligned}
& a_{i} a^{\prime}=0, \\
& u_{0} v=[u, v], u_{1} v=\langle u, v\rangle, \\
& u_{0} a=\pi(u)(a), a_{0} u=-u_{0} a
\end{aligned}
$$

for $a, a^{\prime} \in A, u, v \in B, i=0,1$ such that

$$
\begin{aligned}
& a \cdot\left(a^{\prime} \cdot u\right)-\left(a * a^{\prime}\right) \cdot u=\left(u_{0} a\right) \cdot \partial a^{\prime}+\left(u_{0} a^{\prime}\right) \cdot \partial a, \\
& u_{0}(a \cdot v)-a \cdot\left(u_{0} v\right)=\left(u_{0} a\right) \cdot v, \\
& u_{0}\left(a * a^{\prime}\right)=a *\left(u_{0} a^{\prime}\right)+\left(u_{0} a\right) * a^{\prime}, \\
& a_{0}\left(a^{\prime} \cdot v\right)=a^{\prime} *\left(a_{0} v\right), \\
& (a \cdot u)_{1} v=a *\left(u_{1} v\right)-u_{0} v_{0} a, \\
& \partial\left(a * a^{\prime}\right)=a \cdot \partial\left(a^{\prime}\right)+a^{\prime} \cdot \partial(a) .
\end{aligned}
$$

For the rest of this section, we assume the following:

(i) $(A, *)$ is a unital commutative associative algebra with the identity $\mathfrak{e}$ and $\operatorname{dim}(A)<\infty$.

(ii) $B$ is a vertex $A$-algebroid such that $\operatorname{dim}(B)<\infty$, and $A$ is not a trivial $B$-module.

Recall that a set $I$ is called an ideal of a vertex $A$-algebroid $B$ if $I$ is a left ideal of Leibniz algebra $B$ and $a \cdot u \in I$ for all $a \in A, u \in I$.

Example 14. We set $A \partial(A)=\operatorname{Span}\left\{a \cdot \partial\left(a^{\prime}\right) \mid a, a^{\prime} \in A\right\} . A \partial(A)$ is an ideal of a vertex $A$-algebroid $B$. In fact, $A \partial(A)$ is an abelian Lie algebra.

Proposition 15. [JY] Let $B$ be a simple Leibniz algebra such that Leib $(B) \neq\{0\}$. Assume that its Levi factor $S=\operatorname{Span}\{e, f, h\}$ such that $e_{0} f=h, h_{0} e=2 e, h_{0} f=-2 f$, and $e_{1} f=$ $k \mathfrak{e} \in(\mathbb{C} \mathfrak{e}) \backslash\{0\}$. Then

(i) $e_{1} e=f_{1} f=e_{1} h=f_{1} h=0, k=1, h_{1} h=2 \mathfrak{e}$.

(ii) $\operatorname{Ker}(\partial)=\mathbb{C e}$

(iii) Leib(B) is an irreducible sl ${ }_{2}$-module of dimension 2. Moreover, as a sl ${ }_{2}$-module, $A$ is a direct sum of a trivial module and an irreducible sl $l_{2}$-module of dimension 2.

(iv) $A$ is a local algebra. Let $A_{\neq 0}$ be an irreducible sl $l_{2}$-submodule of $A$ that has dimension 2. Let $a_{0}$ be the highest weight vector of $A_{\neq 0}$ of weight 1 and let $a_{1}=f_{0} a_{0}$. Hence, the set $\left\{a_{0}, a_{1}\right\}$ forms a basis of $A_{\neq 0}$, the set $\left\{\mathfrak{e}, a_{0}, a_{1}\right\}$ is a basis of $A$, and the set $\left\{\partial\left(a_{0}\right), \partial\left(a_{1}\right)\right\}$ is a basis of Leib $(B)$. 
Relationships among $a_{0}, a_{1}, e, f, h, \partial\left(a_{0}\right), \partial\left(a_{1}\right)$ are desribed below:

$$
\begin{aligned}
& \left(\partial\left(a_{0}\right)\right)_{1} e=0, \quad\left(\partial\left(a_{0}\right)\right)_{1} f=a_{1},\left(\partial\left(a_{0}\right)\right)_{1} h=a_{0} \\
& \left(\partial\left(a_{1}\right)\right)_{1} e=a_{0}, \quad\left(\partial\left(a_{1}\right)\right)_{1} f=0,\left(\partial\left(a_{1}\right)\right)_{1} h=-a_{1}, \\
& a_{0} \cdot e=0, a_{0} \cdot f=\partial\left(a_{1}\right), a_{0} \cdot h=\partial\left(a_{0}\right), a_{0} \cdot \partial\left(a_{i}\right)=0 \text { for } i \in\{0,1\} \\
& a_{1} \cdot e=\partial\left(a_{0}\right), a_{1} \cdot f=0, a_{1} \cdot h=-\partial\left(a_{1}\right), a_{1} \cdot \partial\left(a_{i}\right)=0 \text { for } i \in\{0,1\}, \\
& a_{i} * a_{j}=0 \text { for all } i, j \in\{0,1\} .
\end{aligned}
$$

Proposition 16. [JY] Let $B$ be a semisimple Leibniz algebra such that Leib $(B) \neq\{0\}$, and $\operatorname{Ker}(\partial)=\left\{a \in A \mid u_{0} a=0\right.$ for all $\left.u \in B\right\}$.

Assume that the Levi factor $S=\operatorname{Span}\{e, f, h\}$ such that $e_{0} f=h, h_{0} e=2 e, h_{0} f=-2 f$ and $e_{1} f=k \mathfrak{e} \in \mathbb{C} \mathfrak{e} \backslash\{0\}$. We set $A=\mathbb{C} \mathfrak{e} \oplus_{j=1}^{l} N^{j}$ where each $N^{j}$ is an irreducible sl $l_{2}$-submodule of $A$. Then

(i) $e_{1} e=f_{1} f=e_{1} h=f_{1} h=0, k=1, h_{1} h=2 \mathfrak{e}$;

(ii) $\operatorname{Ker}(\partial)=\mathbb{C} \mathfrak{e}$;

(iii) For $j \in\{1, \ldots, l\} \operatorname{dim} N^{j}=2$, and $\operatorname{dim} \operatorname{Leib}(B)=2 l$;

(iv) $A$ is a local algebra. For each $j$, we let $a_{j, 0}$ be a highest weight vector of $N^{j}$ and $a_{j, 1}=$ $f_{0}\left(a_{j, 0}\right)$. Then $\left\{\mathfrak{e}, a_{j, i} \mid j \in\{1, \ldots, l\}, i \in\{0,1\}\right\}$ is a basis of $A$, and $\left\{\partial\left(a_{j, i}\right) \mid j \in\{1, \ldots, l\}, i \in\right.$ $\{0,1\}\}$ is a basis of Leib $(B)$.

Relations among $a_{j, i}, e, f, h, \partial\left(a_{j, i}\right)$ are described below:

$$
\begin{aligned}
& a_{j, i} * a_{j^{\prime}, i^{\prime}}=0, \\
& a_{j, 0} \cdot e=0, a_{j, 1} \cdot e=\partial\left(a_{j, 0}\right), \\
& a_{j, 0} \cdot f=\partial\left(a_{j, 1}\right), a_{j, 1} \cdot f=0, \\
& a_{j, 0} \cdot h=\partial\left(a_{j, 0}\right), a_{j, 1} \cdot h=-\partial\left(a_{j, 1}\right), \\
& a_{j, i} \cdot \partial\left(a_{j^{\prime}, i^{\prime}}\right)=0, \\
& \partial\left(a_{j, i}\right)_{1} e=e_{0} a_{j, i}=(2-i) a_{j, i-1}, \\
& \partial\left(a_{j, i}\right)_{1} f=f_{0} a_{j, i}=(i+1) a_{j, i+1}, \\
& \partial\left(a_{j, i}\right)_{1} h=h_{0} a_{j, i}=(1-2 i) a_{j, i} .
\end{aligned}
$$

\subsection{Vertex Algebras.}

Definition 17. ([Bo1], [FrLMe1], [LLi]) A vertex algebra is a vector space $V$ equipped with a linear map

$$
\begin{aligned}
Y: V & \rightarrow \operatorname{End}(V)\left[\left[x, x^{-1}\right]\right] \\
v & \mapsto Y(v, x)=\sum_{n \in \mathbb{Z}} v_{n} x^{-n-1} \text { where } v_{n} \in \operatorname{End}(V)
\end{aligned}
$$


and equipped with a distinguished vector $\mathbf{1}$, the vacuum vector, such that for $u, v \in V$,

$$
\begin{aligned}
& u_{n} v=0 \text { for } n \text { sufficiently large, } \\
& Y(\mathbf{1}, x)=1, \\
& Y(v, x) \mathbf{1} \in V[[x]], \text { and } \lim _{x \rightarrow 0} Y(v, x) \mathbf{1}=v
\end{aligned}
$$

and such that

$$
\begin{aligned}
& x_{0}^{-1} \delta\left(\frac{x_{1}-x_{2}}{x_{0}}\right) Y\left(u, x_{1}\right) Y\left(v, x_{2}\right)-x_{0}^{-1} \delta\left(\frac{x_{2}-x_{1}}{-x_{0}}\right) Y\left(v, x_{2}\right) Y\left(u, x_{1}\right) \\
& =x_{2}^{-1} \delta\left(\frac{x_{1}-x_{0}}{x_{2}}\right) Y\left(Y\left(u, x_{0}\right) v, x_{2}\right)
\end{aligned}
$$

the Jacobi identity.

From the Jacobi identity we have Borcherds' commutator formula and iterate formula:

$$
\begin{aligned}
& {\left[u_{m}, v_{n}\right]=\sum_{i \geq 0}\left(\begin{array}{c}
m \\
i
\end{array}\right)\left(u_{i} v\right)_{m+n-i}} \\
& \left(u_{m} v\right)_{n} w=\sum_{i \geq 0}(-1)^{i}\left(\begin{array}{c}
m \\
i
\end{array}\right)\left(u_{m-i} v_{n+i} w-(-1)^{m} v_{m+n-i} u_{i} w\right)
\end{aligned}
$$

for $u, v, w \in V, m, n \in \mathbb{Z}$.

We define a linear operator $D$ on $V$ by $D(v)=v_{-2} \mathbf{1}$ for $v \in V$. Then

$$
\begin{aligned}
& Y(v, x) \mathbf{1}=e^{x D} v \text { for } v \in V, \text { and } \\
& {\left[D, v_{n}\right]=(D v)_{n}=-n v_{n-1} \text { for } v \in V, n \in \mathbb{Z} .}
\end{aligned}
$$

Moreover, for $u, v \in V$, we have $Y(u, x) v=e^{x D} Y(v,-x) u$ (skew-symmetry).

A vertex algebra $V$ equipped with a $\mathbb{Z}$-grading $V=\oplus_{n \in \mathbb{Z}} V_{(n)}$ is called a $\mathbb{Z}$-graded vertex algebra if $\mathbf{1} \in V_{(0)}$ and if $u \in V_{(k)}$ with $k \in \mathbb{Z}$ and for $m, n \in \mathbb{Z}, u_{m} V_{(n)} \subseteq V_{(k+n-m-1)}$.

A $\mathbb{N}$-graded vertex algebra is defined in the obvious way.

Proposition 18. GMS

If $V=\oplus_{n \in \mathbb{N}} V_{(n)}$ is an $\mathbb{N}$-graded vertex algebra then

(i) $V_{(0)}$ is a commutative associative algebra with identity 1 and $V_{(1)}$ is Leibniz algebra.

(ii) In fact, $V_{(0)} \oplus V_{(1)}$ is a 1-truncated conformal algebra.

(iii) Moreover, $V_{(1)}$ is a vertex $V_{(0)}$-algebroid.

Proposition 19. [JY] Let $V=\oplus_{n=0}^{\infty} V_{(n)}$ be a $\mathbb{N}$-graded vertex algebra that satisfies the following properties:

(a) $2 \leq \operatorname{dim} V_{(0)}<\infty, 1 \leq \operatorname{dim} V_{(1)}<\infty, V$ is generated by $V_{(0)}$ and $V_{(1)}$;

(b) $V_{(0)}$ is not a trivial module of a Leibniz algebra $V_{(1)}, u_{0} u \neq 0$ for some $u \in V_{(1)}$; 
(c) the Levi factor of $V_{(1)}$ equals Span $\{e, f, h\}, e_{0} f=h, h_{0} e=2 e, h_{0} f=-2 f$ and $e_{1} f=k \mathbf{1}$. Here, $k \in \mathbb{C} \backslash\{0\}$.

Assume that one of the following statements hold.

(i) $V_{(1)}$ is a simple Leibniz algebra;

(ii) $V_{(1)}$ is a semisimple Leibniz algebra and $\operatorname{Ker}(D) \cap V_{(0)}=\left\{a \in V_{(0)} \mid b_{0} a=0\right.$ for all $b \in$ $\left.V_{(1)}\right\}$.

Then $V$ is an indecomposable non-simple vertex algebra.

Definition 20. ([FrLMe1], [LLi]) An ideal of the vertex algebra $V$ is a subspace $I$ such that $u_{n} w \in I$ and $w_{n} u \in I$ for $u \in V, w \in I$ and $n \in \mathbb{Z}$.

Notice that $D(w)=w_{-2} \mathbf{1} \in I$. Hence, under the condition that $D(I) \subseteq I$, the left ideal condition $v_{n} w \in I$ for all $v \in V, w \in I, n \in \mathbb{Z}$ is equivalent to the right ideal condition $w_{m} v \in I$ for all $v \in V, w \in I, m \in \mathbb{Z}$.

For a subset $S$ of a vertex algebra $V$, we denote by $(S)$ the smallest ideal of $V$ containing $S$. It was shown in Corollary 4.5.10 of [LLi] that

$$
(S)=\operatorname{Span}\left\{v_{n} D^{i}(u) \mid v \in V, n \in \mathbb{Z}, i \geq 0, u \in S\right\} .
$$

Definition 21. For a vertex algebra $V$, we define $C_{2}(V)=\operatorname{Span}\left\{u_{-2} v \mid u, v \in V\right\}$. The vertex algebra $V$ is said to satisfy the $C_{2}$-condition if $V / C_{2}(V)$ is finite dimensional

Proposition 22. [Z] For $u, v \in V, n \geq 2, D(v) \in C_{2}(V)$ and $u_{-n} v \in C_{2}(V)$.

Definition 23. LLi] A $V$-module is a vector space $W$ equipped with a linear map $Y_{W}$ from $V$ to $($ End $W)\left[\left[x, x^{-1}\right]\right]$ where $Y_{W}(v, x)=\sum_{n \in \mathbb{Z}} v_{n} x^{-n-1}$ for $v \in V$ such that for $u, v \in V$, $w \in W$,

$$
\begin{aligned}
& u_{n} w=0 \text { for } n \text { sufficiently large, } \\
& Y_{W}(\mathbf{1}, x)=1, \\
& x_{0}^{-1} \delta\left(\frac{x_{1}-x_{2}}{x_{0}}\right) Y_{W}\left(u, x_{1}\right) Y_{W}\left(v, x_{2}\right)-x_{0}^{-1} \delta\left(\frac{x_{2}-x_{1}}{-x_{0}}\right) Y_{W}\left(v, x_{2}\right) Y_{W}\left(u, x_{1}\right) \\
& =x_{2}^{-1} \delta\left(\frac{x_{1}-x_{0}}{x_{2}}\right) Y_{W}\left(Y\left(u, x_{0}\right) v, x_{2}\right) .
\end{aligned}
$$

Definition 24. Let $V=\oplus_{n=0}^{\infty} V_{(n)}$ be a $\mathbb{N}$-graded vertex algebra. A $\mathbb{N}$-graded $V$-module is a $V$-module $M$ equipped with a $\mathbb{N}$-grading $M=\oplus_{n=0}^{\infty} M_{(n)}$ such that $M_{(0)} \neq\{0\}$ and $v_{m} M_{(n)} \subset M_{(n+p-m-1)}$ for $v \in V_{(p)}, p, n \in \mathbb{N}, m \in \mathbb{Z}$.

Proposition 25. LLi Let $V$ be a vertex algebra.

(i) For $v \in V, v$ is weakly nilpotent if and only if $\left(v_{-1}\right)^{r} \mathbf{1}=0$ for some $r>0$.

(ii) Also, if $u \in V$ such that $u_{n} u=0$ for all $n \geq 0$, then $Y\left(\left(u_{-1}\right)^{r} \mathbf{1}, z\right)=Y(u, z)^{r}$ for $r>0$.

(iii) Let $\left(W, Y_{W}\right)$ be a $V$-module. Then for any weakly nilpotent element $v$ of $V$, with $r>0$ chosen so that $\left(v_{-1}\right)^{r} \mathbf{1}=0$, we have $Y_{W}\left(\left(v_{-1}\right)^{r} \mathbf{1}, z\right)=0$. Also for $u \in U$ such that $u_{n} u=0$ for all $n \geq 0$, we have $Y_{W}\left(\left(u_{-1}\right)^{r} \mathbf{1}, z\right)=Y_{W}(u, z)^{r}$ for $r>0$. 
Proposition 26. LiY1 Let $V$ be a vertex algebra and let $I$ be a (two-sided) ideal generated by a subset $S$. Let $\left(W, Y_{W}\right)$ be a $V$-module and let $U$ be a generating subspace of $W$ as a $V$-module such that $Y_{W}(v, x) u=0$ for $v \in S, u \in U$. Then $Y_{W}(v, x)=0$ for $v \in I$.

Next, we recall a construction of vertex algebras associated with vertex algebroids in [LiY1].

Let $A$ be a commutative associative algebra with identity $\mathfrak{e}$ and let $B$ be a vertex $A$-algebroid. We set $L(A \oplus B)=(A \oplus B) \otimes \mathbb{C}\left[t, t^{-1}\right]$. Subspaces $L(A)$ and $L(B)$ of $L(A \oplus B)$ are defined in the obvious way.

We set $\hat{\partial}=\partial \otimes 1+1 \otimes \frac{d}{d t}: L(A) \rightarrow L(A \otimes B)$. We define $\operatorname{deg}\left(a \otimes t^{n}\right)=-n-1, \operatorname{deg}\left(b \otimes t^{n}\right)=-n$ for $a \in A, b \in B, n \in \mathbb{Z}$. Then $L(A \oplus B)$ becomes a $\mathbb{Z}$-graded vector space:

$$
L(A \oplus B)=\oplus_{n \in \mathbb{Z}} L(A \oplus B)_{(n)}
$$

where $L(A \oplus B)_{(n)}=A \otimes \mathbb{C} t^{-n-1}+B \otimes \mathbb{C} t^{-n}$. Clearly, the subspaces $L(A)$ and $L(B)$ are $\mathbb{Z}$-graded vector spaces as well. In addition, for $n \in \mathbb{N}, L(A)_{(n)}=A \otimes \mathbb{C} t^{-n-1}$. The linear map $\hat{\partial}: L(A) \rightarrow L(A \oplus B)$ is of degree 1 . We define a bilinear product $[\cdot, \cdot]$ on $L(A \oplus B)$ as follow:

$$
\begin{aligned}
& {\left[a \otimes t^{m}, a^{\prime} \otimes t^{n}\right]=0,} \\
& {\left[a \otimes t^{m}, b \otimes t^{n}\right]=a_{0} b \otimes t^{m+n},} \\
& {\left[b \otimes t^{n}, a \otimes t^{m}\right]=b_{0} a \otimes t^{m+n},} \\
& {\left[b \otimes t^{m}, b^{\prime} \otimes t^{n}\right]=b_{0} b^{\prime} \otimes t^{m+n}+m\left(b_{1} b\right) \otimes t^{m+n-1}}
\end{aligned}
$$

for $a, a^{\prime} \in A, b, b^{\prime} \in B, m, n \in \mathbb{Z}$. For convenience, we set

$$
\mathcal{L}:=L(A \oplus B) / \hat{\partial} L(A) .
$$

It was shown in [LiY1] that $\mathcal{L}=\oplus_{n \in \mathbb{Z}} \mathcal{L}_{(n)}$ is a $\mathbb{Z}$-graded Lie algebra. Here,

$$
\mathcal{L}_{(n)}=L(A \oplus B)_{(n)} / \hat{\partial}\left(L(A)_{(n-1)}\right)=\left(A \otimes \mathbb{C} t^{-n-1}+B \otimes \mathbb{C} t^{-n}\right) / \hat{\partial}\left(A \otimes \mathbb{C} t^{-n}\right) .
$$

In particular, $\mathcal{L}_{(0)}=A \otimes \mathbb{C} t^{-1}+B / \partial A$.

Let $\rho: L(A \oplus B) \rightarrow \mathcal{L}$ be a natural linear map defined by

$$
\rho\left(u \otimes t^{n}\right)=u \otimes t^{n}+\hat{\partial} L(A) .
$$

For $u \in A \oplus B, n \in \mathbb{Z}$, we set $u(n)=\rho\left(u \otimes t^{n}\right)$ and $u(z)=\sum_{n \in \mathbb{Z}} u(n) z^{-n-1}$. Let $W$ be a $\mathcal{L}$-module. We use $u_{W}(n)$ or sometimes just $u(n)$ for the corresponding operator on $W$ and we write $u_{W}(z)=\sum_{n \in \mathbb{Z}} u(n) z^{-n-1} \in($ End $W)\left[\left[z, z^{-1}\right]\right]$. The commutator relations in terms of generating functions are the following:

$$
\begin{aligned}
& {\left[a\left(z_{1}\right), a^{\prime}\left(z_{2}\right)\right]=0} \\
& {\left[a\left(z_{1}\right), b\left(z_{2}\right)\right]=z_{2}^{-1} \delta\left(\frac{z_{1}}{z_{2}}\right)\left(a_{0} b\right)\left(z_{2}\right)} \\
& {\left[b\left(z_{1}\right), b^{\prime}\left(z_{2}\right)\right]=z_{2}^{-1} \delta\left(\frac{z_{1}}{z_{2}}\right)\left(b_{0} b^{\prime}\right)\left(z_{2}\right)+\left(b_{1} b^{\prime}\right)\left(z_{2}\right) \frac{\partial}{\partial z_{2}} z_{2}^{-1} \delta\left(\frac{z_{1}}{z_{2}}\right)}
\end{aligned}
$$


for $a, a^{\prime} \in A, b, b^{\prime} \in B$.

Next, we define $\mathcal{L}^{\geq 0}=\rho((A \oplus B) \otimes \mathbb{C}[t]) \subset \mathcal{L}$, and $\mathcal{L}^{<0}=\rho\left((A \oplus B) \otimes t^{-1} \mathbb{C}\left[t^{-1}\right]\right) \subset \mathcal{L}$. As a vector space, $\mathcal{L}=\mathcal{L}^{\geq 0} \oplus \mathcal{L}^{<0}$. The subspaces $\mathcal{L}^{\geq 0}$ and $\mathcal{L}^{<0}$ are graded sub-algebras.

We now consider $\mathbb{C}$ as the trivial $\mathcal{L}^{\geq 0}$-module and form the following induced module

$$
V_{\mathcal{L}}=U(\mathcal{L}) \otimes_{U(\mathcal{L} \geq 0)} \mathbb{C} .
$$

In view of the Poincare-Birkhoff-Witt theorem, we have $V_{\mathcal{L}}=U\left(\mathcal{L}^{<0}\right)$ as a vector space. We may consider $A \oplus B$ as a subspace:

$$
A \oplus B \rightarrow V_{\mathcal{L}}, \quad a+b \mapsto a(-1) \mathbf{1}+b(-1) \mathbf{1} .
$$

We assign $\operatorname{deg} \mathbb{C}=0$. Then $V_{\mathcal{L}}=\oplus_{n \in \mathbb{N}}\left(V_{\mathcal{L}}\right)_{(n)}$ is a restricted $\mathbb{N}$-graded $\mathcal{L}$-module. We set $\mathbf{1}=1 \in V_{\mathcal{L}}$.

\section{Proposition 27. FKRW, MeP}

There exists a unique vertex algebra structure on $V_{\mathcal{L}}$ with $Y(u, x)=u(x)$ for $u \in A \oplus B$. In fact, the vertex algebra $V_{\mathcal{L}}$ is a $\mathbb{N}$-graded vertex algebra and it is generated by $A \oplus B$. Furthermore, any restricted $\mathcal{L}$-module $W$ is naturally a $V_{\mathcal{L}}$-module with $Y_{W}(u, x)=u_{W}(x)$ for $u \in A \oplus B$. Conversely, any $V_{\mathcal{L}}$-module $W$ is naturally a restricted $\mathcal{L}$-module with $u_{W}(x)=$ $Y_{W}(u, x)$ for $u \in A \oplus B$.

Now, we set

$$
\begin{aligned}
& E_{0}=\operatorname{Span}\left\{\mathfrak{e}-\mathbf{1}, a(-1) a^{\prime}-a * a^{\prime} \mid a, a^{\prime} \in A\right\} \subset\left(V_{\mathcal{L}}\right)_{(0)}, \\
& E_{1}=\operatorname{Span}\{a(-1) b-a \cdot b \mid a \in A, b \in \mathfrak{L}\} \subset\left(V_{\mathcal{L}}\right)_{(1)}, \\
& E=E_{0} \oplus E_{1}
\end{aligned}
$$

We define

$$
I_{B}=U(\mathcal{L}) \mathbb{C}[D] E .
$$

The vector space $I_{B}$ is an $\mathcal{L}$-submodule of $V_{\mathcal{L}}$. We set

$$
V_{B}=V_{\mathcal{L}} / I_{B}
$$

Proposition 28. GMS, LiY1]

(i) $V_{B}$ is a $\mathbb{N}$-graded vertex algebra such that $\left(V_{B}\right)_{(0)}=A$ and $\left(V_{B}\right)_{(1)}=B$ (under the linear map $v \mapsto v(-1) \mathbf{1})$ and $V_{B}$ as a vertex algebra is generated by $A \oplus B$. Furthermore, for any $n \geq 1$,

$$
\begin{aligned}
& \left(V_{B}\right)_{(n)} \\
& =\operatorname{span}\left\{b_{1}\left(-n_{1}\right) \ldots . . b_{k}\left(-n_{k}\right) \mathbf{1} \mid b_{i} \in B, n_{1} \geq \ldots \geq n_{k} \geq 1, n_{1}+\ldots+n_{k}=n\right\} .
\end{aligned}
$$

(ii) $A V_{B}$-module $W$ is a restricted module for the Lie algebra $\mathcal{L}$ with $v(n)$ acting as $v_{n}$ for $v \in A \oplus B, n \in \mathbb{Z}$. Furthermore, the set of $V_{B}$-submodules is precisely the set of $\mathcal{L}$-submodules.

Next, we recall definition of Lie algebroid and its module. Also, we will review construction of $V_{B}$-modules from modules of Lie $A$-algebroid $B / A \partial(A)$. 
Definition 29. Let $A$ be a commutative associative algebra. A Lie A-algebroid is a Lie algebra $\mathfrak{g}$ equipped with an $A$-module structure and a module action on $A$ by derivation such that

$$
[u, a v]=a[u, v]+(u a) v, \quad a(u b)=(a u) b
$$

for all $u, v \in \mathfrak{g}, a, b \in A$.

A module for a Lie A-algebroid $\mathfrak{g}$ is a vector space $W$ equipped with a $\mathfrak{g}$-module structure and an $A$-module structure such that

$$
u(a w)-a(u w)=(u a) w, a(u w)=(a u) w
$$

for $a \in A, u \in \mathfrak{g}, w \in W$.

Proposition 30. [LiY1] Let $W=\oplus_{n \in \mathbb{N}} W_{(n)}$ be a $\mathbb{N}$-graded $V_{B}$-module with $W_{(0)} \neq\{0\}$. Then $W_{(0)}$ is an $A$-module with $a \cdot w=a_{-1} w$ for $a \in A, w \in W_{(0)}$ and $W_{(0)}$ is a module for the Lie algebra $B / A \partial(A)$ with $b \cdot w=b_{0} w$ for $b \in B, w \in W_{(0)}$. Furthermore, $W_{(0)}$ equipped

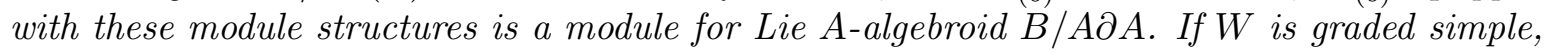
then $W_{(0)}$ is a simple module for Lie A-algebroid $B / A \partial A$.

Now, we set $\mathcal{L}_{ \pm}=\oplus_{n \geq 1} \mathcal{L}_{( \pm n)}$ and $\mathcal{L}_{\leq 0}=\mathcal{L}_{-} \oplus \mathcal{L}_{(0)}$. Let $U$ be a module for the Lie algebra $\mathcal{L}_{(0)}(=A \oplus B / \partial(A))$. Then $U$ is an $\overline{\mathcal{L}}_{(\leq 0)}$-module under the following actions:

$$
a(n-1) \cdot u=\delta_{n, 0} a u, \quad b(n) \cdot u=\delta_{n, 0} u \text { for } a \in A, b \in B, n \geq 0 .
$$

Next, we form the induced $\mathcal{L}$-module $M(U)=\operatorname{Ind}_{\mathcal{L}_{(\leq 0)}}^{\mathcal{L}} U$. Endow $U$ with degree 0, making $M(U)$ a $\mathbb{N}$-graded $\mathcal{L}$-module. In fact, $M(U)$ is a $V_{\mathcal{L}}$-module. We set

$$
W(U)=\operatorname{span}\left\{v_{n} u \mid v \in E, n \in \mathbb{Z}, u \in U\right\} \subset M(U),
$$

and

$$
M_{B}(U)=M(U) / U(\mathcal{L}) W(U)
$$

Proposition 31. LiY1]

(i) Let $U$ be a module for the Lie algebra $\mathcal{L}_{(0)}$. Then $M_{B}(U)$ is a $V_{B}$-module. If $U$ is a module for the Lie A-algebroid $B / A \partial A$ then $\left(M_{B}(U)\right)_{(0)}=U$.

(ii) Let $U$ be a module for the Lie A-algebroid $B / A \partial A$. Then there exists a unique maximal graded $U(\mathcal{L})$-submodule $J(U)$ of $M(U)$ with the property that $J(U) \cap U=0$. Moreover, $L(U)=M(U) / J(U)$ is a $\mathbb{N}$-graded $V_{B}$-module such that $L(U)_{(0)}=U$ as a module for the Lie $A$-algebroid $B / A \partial A$. If $U$ is a simple $B / A \partial A, L(U)$ is a graded simple $V_{B}$-module.

(iii) Let $W=\coprod_{n \in \mathbb{N}} W_{(n)}$ be an $\mathbb{N}$-graded simple $V_{B}$-module with $W_{(0)} \neq 0$. Then $W \cong$ $L\left(W_{(0)}\right)$.

(iv) For any complete set $H$ of representatives of equivalence classes of simple modules for the

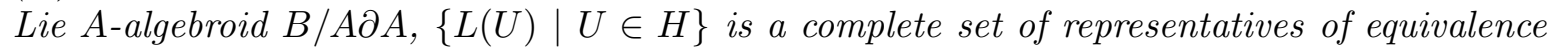
classes of simple $\mathbb{N}$-graded simple $V_{B}$-modules. 


\section{Proof of Theorem 1 and Theorem 2}

Let $A$ be a finite-dimensional commutative associative algebra with the identity $\mathfrak{e}$ such that $\operatorname{dim} A \geq 2$. Let $B$ be a finite-dimensional vertex $A$-algebroid such that $A$ is not a trivial $B$-module and $\operatorname{Leib}(B) \neq\{0\}$. Let $S$ be its Levi factor such that $S=\operatorname{Span}\{e, f, h\}, e_{0} f=h$, $h_{0} e=2 e, h_{0} f=-2 f$, and $e_{1} f=k \mathfrak{e}$. Here, $k \in \mathbb{C} \backslash\{0\}$. Assume that one of the following statements hold.

(I) $B$ is a simple Leibniz algebra;

(II) $B$ is a semisimple Leibniz algebra and $\operatorname{Ker}(\partial)=\left\{a \in A \mid b_{0} a=0\right.$ for all $\left.b \in B\right\}$.

We set $A=\mathbb{C e}_{\mathfrak{e}} \oplus_{j=1}^{l} N^{j}$ where each $N^{j}$ is an irreducible $s l_{2}$-submodule of $A$. By Proposition 15] and Proposition [16, we have

(i) $e_{1} e=f_{1} f=e_{1} h=f_{1} h=0, k=1, h_{1} h=2 \mathfrak{e}$;

(ii) $\operatorname{Ker}(\partial)=\mathbb{C} \mathfrak{e}$ and $l \geq 1$;

(iii) For $j \in\{1, \ldots, l\} \operatorname{dim} N^{j}=2$, and $\operatorname{dim} \operatorname{Leib}(B)=2 l$;

(iv) $A$ is a local algebra. For each $j$, we let $a_{j, 0}$ be a highest weight vector of $N^{j}$ and $a_{j, 1}=f_{0}\left(a_{j, 0}\right)$. Then $\left\{\mathfrak{e}, a_{j, i} \mid j \in\{1, \ldots, l\}, i \in\{0,1\}\right\}$ is a basis of $A$, and $\left\{\partial\left(a_{j, i}\right) \mid j \in\right.$ $\{1, \ldots, l\}, i \in\{0,1\}\}$ is a basis of $\operatorname{Leib}(B)$.

Relations among $a_{j, i}, e, f, h, \partial\left(a_{j, i}\right)$ are described below:

$$
\begin{aligned}
& a_{j, i} * a_{j^{\prime}, i^{\prime}}=0, a_{j, 0} \cdot e=0, a_{j, 1} \cdot e=\partial\left(a_{j, 0}\right), a_{j, 0} \cdot f=\partial\left(a_{j, 1}\right), a_{j, 1} \cdot f=0, \\
& a_{j, 0} \cdot h=\partial\left(a_{j, 0}\right), a_{j, 1} \cdot h=-\partial\left(a_{j, 1}\right), a_{j, i} \cdot \partial\left(a_{j^{\prime}, i^{\prime}}\right)=0, \\
& \partial\left(a_{j, i}\right)_{1} e=e_{0} a_{j, i}=(2-i) a_{j, i-1}, \partial\left(a_{j, i}\right)_{1} f=f_{0} a_{j, i}=(i+1) a_{j, i+1}, \\
& \partial\left(a_{j, i}\right)_{1} h=h_{0} a_{j, i}=(1-2 i) a_{j, i} .
\end{aligned}
$$

\subsection{Proof of Theorem 1 .}

First, we will prove statement $(i)$ of Theorem 1 .

Lemma 32. $V_{B}$ is an indecomposable non-simple vertex algebra.

Proof. Recall that $\left(V_{B}\right)_{(0)}=A,\left(V_{B}\right)_{(1)}=B$ and $V_{B}$ is generated by $A$ and $B$. By Proposition [19. we can conclude that $V_{B}$ is an indecomposable non-simple vertex algebra.

Now, we prove statement $(i i)$ of Theorem 1. First, we show in Lemma 33 that if $U$ is an irreducible $B / A \partial(A)$-module such that $a_{j, i}$ acts as zero for all $j \in\{1, \ldots, l\}, i \in\{0,1\}$ then $U$ is an irreducible module for the Lie $A$-algebroid $B / A \partial(A)$. Next, we prove in Lemma 34 that the converse of the previous statement holds when $U$ has finite dimension (i.e., if $U$ is a finite dimensional irreducible module for the Lie $A$-algebroid $B / A \partial(A)$ then $a_{j, i}$ acts trivial on $U$ for all $j \in\{1, \ldots, l\}, i \in\{0,1\}$.) We complete the proof of the statement (ii) of Theorem 1 in Lemma 35. 
Lemma 33. Let $U$ be an irreducible $B / A \partial(A)$-module. If $\mathfrak{e}$ acts as a scalar 1 , and $a_{j, i}$ acts trivially on $U$ for all $j \in\{1, \ldots, l\}, i \in\{0,1\}$ then $U$ is an irreducible module for a Lie A-algebroid $B / A \partial(A)$.

Proof. Let $U$ be an irreducible $B / A \partial(A)$-module. Assume that $\mathfrak{e}$ acts as a scalar 1 , and $a_{j, i}$ acts trivially on $U$ for all $j \in\{1, \ldots, l\}, i \in\{0,1\}$. First, we will show that $U$ is a module for the associative algebra $A$. Let $a=\beta \mathfrak{e}+\sum_{j=1}^{l} \sum_{i=0}^{1} \lambda_{j, i} a_{j, i}, a^{\prime}=\beta^{\prime} \mathfrak{e}+\sum_{j=1}^{l} \sum_{i=0}^{1} \lambda_{j, i}^{\prime} a_{j, i} \in A$. Here, $\beta, \beta^{\prime}, \lambda_{j, i}, \lambda_{j, i}^{\prime} \in \mathbb{C}$. Since

$$
\begin{aligned}
\left(a * a^{\prime}\right) \cdot w & =\left(\left(\beta \mathfrak{e}+\sum_{j=1}^{l} \sum_{i=0}^{1} \lambda_{j, i} a_{j, i}\right) *\left(\beta^{\prime} \mathfrak{e}+\sum_{j=1}^{l} \sum_{i=0}^{1} \lambda_{j, i}^{\prime} a_{j, i}\right)\right) \cdot w \\
& =\left(\beta a^{\prime}+\beta^{\prime} \sum_{j=1}^{l} \sum_{i=0}^{1} \lambda_{j, i} a_{j, i}\right) \cdot w=\left(\beta \beta^{\prime}\right) w, \text { and } \\
a \cdot\left(a^{\prime} \cdot w\right) & =a \cdot\left(\beta^{\prime} w\right)=\beta \beta^{\prime} w \text { for all } w \in U,
\end{aligned}
$$

we can conclude that $U$ is a module for the associative algebra $A$.

Now, we will show that $U$ is a module for the Lie $A$-algebroid $B / A \partial(A)$. It is enough to show that for $a \in A, u \in B / A \partial(A), w \in U, u_{0}(a \cdot w)-a \cdot\left(u_{0} w\right)=\left(u_{0} a\right) \cdot w$ and $a \cdot\left(u_{0} w\right)=(a \cdot u)_{0} w$. Recall that for $a, a^{\prime} \in A, b \in B,(a \cdot v)_{0} a^{\prime}=-a_{0}^{\prime}(a \cdot v)=-a *\left(a_{0}^{\prime} v\right)=a *\left(v_{0} a^{\prime}\right)$. Consequently,

$$
\left(\alpha \cdot \partial\left(\alpha^{\prime}\right)\right)_{0} a^{\prime}=\alpha *\left(\partial\left(\alpha^{\prime}\right)_{0} a^{\prime}\right)=0 \text { for all } \alpha, \alpha^{\prime}, a^{\prime} \in A .
$$

We let $u=\gamma_{e} e+\gamma_{f} f+\gamma_{h} h+A \partial(A) \in B / A \partial(A)$. Here, $\gamma_{e}, \gamma_{f}, \gamma_{h} \in \mathbb{C}$. Observe that

$$
\begin{aligned}
u_{0} a & =\left(\gamma_{e} e+\gamma_{f} f+\gamma_{h} h+A \partial(A)\right)_{0}\left(\beta \mathfrak{e}+\sum_{j=1}^{l} \sum_{i=0}^{1} \lambda_{j, i} a_{j, i}\right) \\
& =\gamma_{e} \sum_{j=1}^{l} \lambda_{j, 1} a_{j, 0}+\gamma_{f} \sum_{j=1}^{l} \lambda_{j, 0} a_{j, 1}+\gamma_{h} \sum_{j=1}^{l} \lambda_{j, 0} a_{j, 0}+\gamma_{h} \sum_{j=1}^{l} \lambda_{j, 1}\left(-a_{j, 1}\right) .
\end{aligned}
$$

Hence, $\left(u_{0} a\right) \cdot w=0$ for all $w \in U$. Since

$$
\begin{aligned}
u_{0}(a \cdot w)-a \cdot\left(u_{0} w\right) & =u_{0}\left(\left(\beta \mathfrak{e}+\sum_{j=1}^{l} \sum_{i=0}^{1} \lambda_{j, i} a_{j, i}\right) \cdot w\right)-\left(\beta \mathfrak{e}+\sum_{j=1}^{l} \sum_{i=0}^{1} \lambda_{j, i} a_{j, i}\right) \cdot\left(u_{0} w\right) \\
& =u_{0}(\beta w)-\beta\left(u_{0} w\right) \\
& =0
\end{aligned}
$$

we can conclude immediately that

$$
u_{0}(a \cdot w)-a \cdot\left(u_{0} w\right)=\left(u_{0} a\right) \cdot w \text { for all } w \in U .
$$

Recall that for $j \in\{1, \ldots, l\}, i \in\{0,1\}$, we have $a_{j, i} \cdot v \in \partial(A)$ for all $v \in B$. It follows that

$$
(a \cdot u)_{0} w=(\beta u)_{0} w=\beta u_{0} w \text { for all } w \in U .
$$


Moreover, we have

$$
a \cdot\left(u_{0} w\right)=\beta\left(u_{0} w\right)=(a \cdot u)_{0} w \text { for all } w \in U .
$$

Therefore, $U$ is a module for the Lie $A$-algebroid $B / A \partial(A)$.

Next, we will show that $U$ is an irreducible module for the Lie $A$-algebroid $B / A \partial(A)$. Let $N$ be a nonzero Lie $A$-algebroid $B / A \partial(A)$-submodule of $U$. Then $N$ is a $B / A \partial(A)$-submodule of $U$. Since $U$ is an irreducible $B / A \partial(A)$, we can conclude that $N=U$ and $U$ is an irreducible module for the Lie $A$-algebroid $B / A \partial(A)$. This completes the proof of this Lemma.

Lemma 34. Let $W$ be a finite dimensional irreducible module of the Lie A-algebroid $B / A \partial(A)$. Then for $j \in\{1, \ldots, l\}, i \in\{0,1\}, a_{j, i}$ acts trivially on $W$. In addition, $W$ is an irreducible $s_{2}$-module.

Proof. Let $W$ be a finite dimensional irreducible module of the Lie $A$-algebroid $B / A \partial(A)$. First, we will show that if $W$ has dimension 1 then $W$ is a trivial $s l_{2}$-module such that for $j \in\{1, \ldots, l\}, i \in\{0,1\}, a_{j, i}$ acts as zero on $W$. For simplicity, we assume that $W=\mathbb{C} b$ for some $b \in W$. Clearly, $W$ is a trivial $s l_{2}$-module. We set $a_{j, i} \cdot b=\beta_{j, i} b$. Here, $\beta_{j, i} \in \mathbb{C}$. Since

$$
\begin{aligned}
& h_{0}\left(a_{j, i} \cdot b\right)=\left(h_{0} a_{j, i}\right) \cdot b+\left(a_{j, i}\right) \cdot\left(h_{0} b\right)=\left(h_{0} a_{j, i}\right) \cdot b, \text { and } \\
& h_{0}\left(a_{j, i} \cdot b\right)=h_{0}\left(\beta_{j, i} b\right)=0,
\end{aligned}
$$

we then have that

$$
\begin{aligned}
& 0=\left(h_{0} a_{j, 0}\right) \cdot b=a_{j, 0} \cdot b=\beta_{j, 0} b \text { and } \\
& 0=\left(h_{0} a_{j, 1}\right) \cdot b=-a_{j, 1} \cdot b=-\beta_{j, 1} b .
\end{aligned}
$$

Therefore, $\beta_{j, i}=0$ and $a_{j, i}$ acts as zero on $W$ for all $j \in\{1, \ldots, l\}$ and $i \in\{0,1\}$.

Next, we assume that $W$ has dimension 2. Hence, $W$ is either a direct sum of two onedimensional trivial $s l_{2}$-modules or $W$ is a two-dimensional irreducible $s l_{2}$-module. Suppose that $W=\mathbb{C} b_{1} \oplus \mathbb{C} b_{2}$ where $\mathbb{C} b_{1}$ and $\mathbb{C} b_{2}$ are trivial $B / A \partial(A)$-modules. For $j \in\{1, \ldots, l\}$, $i \in\{0,1\}$, we set $a_{j, i} \cdot b_{1}=\beta_{j, i, 1} b_{1}+\beta_{j, i, 2} b_{2}$. Since

$$
h_{0}\left(a_{j, i} \cdot b_{1}\right)=\left(h_{0}\left(a_{j, i}\right)\right) \cdot b_{1} \text { and } h_{0}\left(\beta_{j, i, 1} b_{1}+\beta_{j, i, 2} b_{2}\right)=0 \text {, }
$$

we then have that $a_{j, i} \cdot b_{1}=0$ for all $j \in\{1, \ldots, l\}, i \in\{0,1\}$. Consequently, $\mathbb{C} b_{1}$ is an irreducible $A$-Lie algebroid $B / A \partial(A)$. This contradicts with our assumption that $W$ is an irreducible Lie $A$-algebroid $B / A \partial(A)$. Therefore, $W$ is a two-dimensional irreducible $s l_{2}$-module. Let $w_{0}$ be a highest weight vector of $W$ of weight 1 and let $w_{1}=f_{0}\left(w_{0}\right)$. The set $\left\{w_{0}, w_{1}\right\}$ is a basis of $W$. For $j \in\{1, \ldots, l\}, i \in\{0,1\}$, we set

$$
a_{j, i} \cdot\left(w_{0}\right)=\alpha_{j, i, 0} w_{0}+\alpha_{j, i, 1} w_{1} .
$$

Here, $\alpha_{j, i, 0}, \alpha_{j, i, 1} \in \mathbb{C}$. Notice that

$$
\begin{aligned}
& h_{0}\left(a_{j, i} \cdot w_{0}\right)=\left(h_{0} a_{j, i}\right) \cdot w_{0}+a_{j, i} \cdot\left(h_{0}\left(w_{0}\right)\right)=\left(h_{0} a_{j, i}\right) \cdot w_{0}+a_{j, i} \cdot w_{0}, \text { and } \\
& h_{0}\left(\alpha_{j, i, 0} w_{0}+\alpha_{j, i, 1} w_{1}\right)=\alpha_{j, i, 0} w_{0}+\alpha_{j, i, 1}\left(-w_{1}\right) .
\end{aligned}
$$


So, we have

$$
\begin{aligned}
& 2 a_{j, 0} \cdot w_{0}=\left(h_{0} a_{j, 0}\right) \cdot w_{0}+a_{j, 0} \cdot w_{0}=\alpha_{j, 0,0} w_{0}+\alpha_{j, 0,1}\left(-w_{1}\right), \text { and } \\
& 0=\left(h_{0} a_{j, 1}\right) \cdot w_{0}+a_{j, 1} \cdot w_{0}=\alpha_{j, 1,0} w_{0}+\alpha_{j, 1,1}\left(-w_{1}\right) .
\end{aligned}
$$

Therefore, for $j \in\{1, \ldots, l\}, i \in\{0,1\}$, we have $\alpha_{j, i, 0}=\alpha_{j, i, 1}=0$ and $a_{j, i} \cdot w_{0}=0$. Since $a_{j, 0} \cdot f=\partial\left(a_{j, 1}\right)$ and $a_{j, 1} \cdot f=0$, these imply that

$$
a_{j, i} \cdot w_{1} \quad=a_{j, i} \cdot\left(f_{0} w_{0}\right)=\left(a_{j, i} \cdot f\right)_{0}\left(w_{0}\right)=0
$$

Consequently, if $\operatorname{dim} W=2$ then $W$ is an irreducible $B / A \partial(A)$-module such that for $j \in$ $\{1, \ldots, l\}, i \in\{0,1\}, a_{j, i}$ acts trivially on $W$.

Now, we study that case when $\operatorname{dim} W \geq 3$. Suppose that $W$ contains a nonzero proper $B / A \partial(A)$-submodule (i.e., we consider $W$ as a module for the Lie algebra $B / A \partial(A))$. Since $B / A \partial(A)$ is semisimple, this implies that there exist irreducible $B / A \partial(A)$-modules $U_{1}, \ldots, U_{t}$ such that $W=\oplus_{i=1}^{t} U_{t}$. For each $i \in\{1, \ldots, t\}$, we let $w_{i, 0}$ be a highest weight vector of $U_{i}$ of weight $m_{i}$. Also, we set $w_{i, s}=\frac{1}{s !}(f(0))^{s} w_{i, 0}$. Clearly, $\left\{w_{i, 0}, \ldots ., w_{i, m_{i}}\right\}$ form a basis of $U_{i}$. Let $j \in\{1, \ldots, l\}, 1 \leq s \leq m_{i}$. Since $a_{j, 0} \cdot f=\partial\left(a_{j, 1}\right)$ and $a_{j, 1} \cdot f=0$, we then have that

$$
\begin{aligned}
a_{j, 0} \cdot w_{i, s} & =a_{j, 0} \cdot\left(\frac{1}{s !}\left(f_{0}\right)^{s} w_{i, 0}\right)=a_{j, 0} \cdot\left(f_{0}\left(\frac{1}{s !}\left(f_{0}\right)^{s-1} w_{i, 0}\right)\right) \\
& =\left(a_{j, 0} \cdot f\right)_{0}\left(\frac{1}{s !}\left(f_{0}\right)^{s-1} w_{i, 0}\right)=0
\end{aligned}
$$

and

$$
a_{j, 1} \cdot w_{i, s}=a_{j, 1} \cdot\left(\frac{1}{s !}\left(f_{0}\right)^{s} w_{i, 0}\right)=\left(a_{j, 1} \cdot f\right)_{0}\left(\frac{1}{s !}\left(f_{0}\right)^{s-1} w_{i, 0}\right)=0
$$

Since $h_{0}\left(a_{j, 0} \cdot w_{i, 0}\right)=\left(h_{0} a_{j, 0}\right) \cdot w_{i, 0}+a_{j, 0} \cdot\left(h_{0} w_{i, 0}\right)=\left(m_{i}+1\right) a_{j, 0} \cdot w_{i, 0}$ and

$$
\begin{aligned}
h_{0}\left(a_{j, 0} \cdot w_{i, 0}\right) & =e_{0} f_{0}\left(a_{j, 0} \cdot w_{i, 0}\right)-f_{0} e_{0}\left(a_{j, 0} \cdot w_{i, 0}\right) \\
& =e_{0}\left(f_{0}\left(a_{j, 0}\right) \cdot w_{i, 0}+a_{j, 0} \cdot\left(f_{0} w_{i, 0}\right)\right)-f_{0}\left(\left(e_{0} a_{j, 0}\right) \cdot w_{i, 0}+a_{j, 0} \cdot e_{0} w_{i, 0}\right) \\
& =e_{0}\left(a_{j, 1} \cdot w_{i, 0}+a_{j, 0} \cdot w_{i, 1}\right) \\
& =e_{0}\left(a_{j, 1} \cdot w_{i, 0}\right) \\
& =\left(e_{0} a_{j, 1}\right) \cdot w_{i, 0}+a_{j, 1} \cdot\left(e_{0} w_{i, 0}\right) \\
& =a_{j, 0} \cdot w_{i, 0}
\end{aligned}
$$

we can conclude that $m_{i}=0$ and $U_{i}$ is a trivial $B / A \partial(A)$-module. Moreover, $\left\{w_{1,0}, \ldots, w_{t, 0}\right\}$ is a basis of $W$. For $j \in\{1, \ldots, l\}, i \in\{1, \ldots, t\}$, we set $a_{j, 0} \cdot w_{i, 0}=\sum_{p=1}^{t} \alpha_{p} w_{p, 0}$, and 
$a_{j, 1} \cdot w_{i, 0}=\sum_{p=1}^{t} \gamma_{p} w_{p, 0}$ where $\alpha_{p}, \gamma_{p} \in \mathbb{C}$. Since

$$
\begin{aligned}
& h_{0}\left(a_{j, 0} \cdot w_{i, 0}\right)=\left(h_{0} a_{j, 0}\right) \cdot w_{i, 0}+a_{j, 0} \cdot h_{0} w_{i, 0}=a_{j, 0} \cdot w_{i, 0}, \\
& h_{0}\left(\sum_{p=1}^{t} \alpha_{p} w_{p, 0}\right)=0, \\
& h_{0}\left(a_{j, 1} \cdot w_{i, 0}\right)=\left(h_{0} a_{j, 1}\right) \cdot w_{i, 0}+a_{j, 1} \cdot h_{0} w_{i, 0}=-a_{j, 1} \cdot w_{i, 0}, \\
& h_{0}\left(\sum_{p=1}^{t} \gamma_{p} w_{p, 0}\right)=0,
\end{aligned}
$$

we can conclude that $a_{j, 0} \cdot w_{i, 0}=0=a_{j, 1} \cdot w_{i, 0}$ for all $j \in\{1, \ldots, l\}, i \in\{1, \ldots, t\}$. Moreover, each $U_{i}$ is an irreducible module for the Lie $A$-algebroid $B / A \partial(A)$. This is a contradiction. Hence, $W$ is an irreducible $B / A \partial(A)$-module.

Now, we let $u_{0}$ be the highest weight vector of $W$ with weight $m$ and for $i \in\{1, \ldots, m\}$ we let $u_{i}=\frac{1}{i !}(f(0))^{i} u_{0}$. We have

$$
a_{j, 0} \cdot u_{i}=a_{j, 0} \cdot\left(\frac{1}{i !}\left(f_{0}\right)^{i} u_{0}\right)=a_{j, 0} \cdot\left(f_{0}\left(\frac{1}{i !}\left(f_{0}\right)^{i-1} u_{0}\right)\right)=\left(a_{j, 0} \cdot f\right)_{0}\left(\frac{1}{i !}\left(f_{0}\right)^{i-1} u_{0}\right)=0,
$$

and

$$
a_{j, 1} \cdot u_{i}=a_{j, 1} \cdot\left(\frac{1}{i !}\left(f_{0}\right)^{i} u_{0}\right)=\left(a_{j, 1} \cdot f\right)_{0}\left(\frac{1}{i !}\left(f_{0}\right)^{i-1} u_{0}\right)=0 .
$$

Next, we set $a_{j, 0} \cdot u_{0}=\sum_{q=0}^{m} \alpha_{q} u_{q}$. Since

$$
\begin{aligned}
& h_{0}\left(a_{j, 0} \cdot u_{0}\right)=(m+1)\left(a_{j, 0} \cdot u_{0}\right)=\sum_{q=0}^{m} \alpha_{q}(m+1) u_{q} \text { and } \\
& h_{0}\left(\sum_{q=0}^{m} \alpha_{q} u_{q}\right)=\sum_{q=0}^{m}(m-2 q) \alpha_{q} u_{q},
\end{aligned}
$$

we can conclude that $\alpha_{q}(m+1)=\alpha_{q}(m-2 q)$ for all $0 \leq q \leq m$. If $\alpha_{q} \neq 0$, we have $m+1=m-2 q$ which is impossible. Therefore, for all $0 \leq q \leq m, \alpha_{q}=0$. Consequently, we have $a_{j, 0} \cdot u_{0}=0$. Moreover, we have

$$
a_{j, 1} \cdot u_{0}=\left(f_{0} a_{j, 0}\right) \cdot u_{0}=f_{0}\left(a_{j, 0} \cdot u_{0}\right)-a_{j, 0} \cdot\left(f_{0} u_{0}\right)=-a_{j, 0} \cdot u_{1}=0 .
$$

Hence, $W$ is an irreducible $B / A \partial(A)$-module such that for $j \in\{1, \ldots, l\}, i \in\{0,1\}, a_{j, i}$ acts trivially on $W$. This completes the proof of this Lemma.

Lemma 35. The set of representatives of equivalence classes of finite-dimensional simple sl $2_{2}-$ modules is equivalent to the set of representatives of equivalence classes of $\mathbb{N}$-graded simple $V_{B}$-modules $N=\oplus_{n=0}^{\infty} N_{(n)}$ such that $\operatorname{dim} N_{(0)}<\infty$.

Proof. By Lemma 33, every finite dimensional irreducible $B / A \partial(A)$-module is an irreducible module for the Lie $A$-algebroid $B / A \partial(A)$. By Lemma 34, the set of representatives of equivalence classes of finite dimensional simple modules for the Lie $A$-algebraoid $B / A \partial(A)$ equals the set of representatives of equivalence classes of finite dimensional simple modules for the 
Lie algebra $B / A \partial(A)$. By Proposition 31, we can conclude that the set of representatives of equivalence classes of finite-dimensional simple $s l_{2}$-modules is equivalent to the set of representatives of equivalence classes of $\mathbb{N}$-graded simple $V_{B}$-modules $N=\oplus_{n=0}^{\infty} N_{(n)}$ such that $\operatorname{dim} N_{(0)}<\infty$.

This completes the proof of statment $(i i)$ of Theorem 1 .

\subsection{Proof of Theorem 2 .}

First, we will prove statement $(i)$ of Theorem 2, Let $(e(-1) e)$ be an ideal of $V_{B}$ that is generated by $e(-1) e$.

Lemma 36. $(e(-1) e) \cap A=\{0\}$ and $(e(-1) e) \cap B=\{0\}$.

Proof. First, we will show that $v_{(\operatorname{deg} v)+1} e(-1) e=0$ and $v_{\operatorname{deg} v} e(-1) e=0$ for every homogeneous $v \in V_{B}$. We will separate our proof into several steps. For the first step, we will show that for $a \in A$,

$$
a(n) e(-1) e=0 \text { for all } n \geq 0 .
$$

Recall that for $a \in A, b \in B$, we have $b(-1) a=a(-1) b-D(a(0) b)=a \cdot b-D\left(a_{0} b\right)$. Let $a=\alpha \mathbf{1}+\sum_{j=1}^{l} \sum_{i=0}^{1} \alpha_{j, i} a_{j, i}$. Here, $\alpha, \alpha_{j, i} \in \mathbb{C}$. It is straightforward to show that

$$
\begin{aligned}
& a(0) e(-1) e \\
& =e(-1) a_{0} e+\left(a_{0} e\right)(-1) e \\
& =-e(-1) e_{0} a-\left(e_{0} a\right)(-1) e \\
& =-e(-1)\left(\sum_{j=1}^{l} \alpha_{j, 1} a_{j, 0}\right)-\sum_{j=1}^{l} \alpha_{j, 1}\left(a_{j, 0}\right)(-1) e \\
& =-e(-1)\left(\sum_{j=1}^{l} \alpha_{j, 1} a_{j, 0}\right)-\sum_{j=1}^{l} \alpha_{j, 1}\left(a_{j, 0}\right) \cdot e \\
& =-\left(\sum_{j=1}^{l} \alpha_{j, 1} a_{j, 0}\right) \cdot e+D\left(\left(\sum_{j=1}^{l} \alpha_{j, 1} a_{j, 0}\right)_{0} e\right) \\
& =0,
\end{aligned}
$$

and $a(1) e(-1) e=e(-1) a(1) e+\left(a_{0} e\right)(0) e=e_{0}\left(e_{0} a\right)=e_{0}\left(\sum_{j=1}^{l} \alpha_{j, 1} a_{j, 0}\right)=0$. Hence,

$$
a(n) e(-1) e=0 \text { for all } n \geq 0 .
$$

For the second step, we will show that for $b \in B$,

$$
(b(-1) \mathbf{1})_{n} e(-1) e=b(n) e(-1) e=0,(b(-m) \mathbf{1})_{m+1} e(-1) e=0, \text { and }(b(-m) \mathbf{1})_{m} e(-1) e=0
$$


for all $m \geq 2, n \geq 1$. Let $b=\beta_{e} e+\beta_{f} f+\beta_{h} h+\sum_{j=1}^{l} \sum_{i=0}^{1} \beta_{j, i} \partial\left(a_{j, i}\right) \in B$. Here, $\beta_{e}, \beta_{f}, \beta_{h}, \beta_{j, i} \in \mathbb{C}$. Since

$$
\begin{aligned}
b_{0} e \quad & =\left(\beta_{e} e+\beta_{f} f+\beta_{h} h+\sum_{j=1}^{l} \sum_{i=0}^{1} \beta_{j, i} \partial\left(a_{j, i}\right)\right)_{0} e \\
& =\beta_{f}(-h)+\beta_{h}(2 e) \text { and } \\
b_{1} e \quad & =\left(\beta_{e} e+\beta_{f} f+\beta_{h} h+\sum_{j=1}^{l} \sum_{i=0}^{1} \beta_{j, i} \partial\left(a_{j, i}\right)\right)_{1} e \\
& =\beta_{f} \mathbf{1}+\sum_{j=1}^{l} \beta_{j, 1} a_{j, 0}
\end{aligned}
$$

we then have that

$$
\begin{aligned}
b(1) e(-1) e \quad & =e(-1) b(1) e+\left(b_{0} e\right)(0) e+\left(b_{1} e\right)(-1) e \\
& =e(-1)\left(b_{1} e\right)+\left(\beta_{f}(-h)+\beta_{h}(2 e)\right)_{0} e+\left(\beta_{f} \mathbf{1}+\sum_{j=1}^{l} \beta_{j, 1} a_{j, 0}\right)(-1) e \\
& =e(-1)\left(\beta_{f} \mathbf{1}+\sum_{j=1}^{l} \beta_{j, 1} a_{j, 0}\right)+\beta_{f}(-2) e+\beta_{f} e \\
& =\sum_{j=1}^{l} \beta_{j, 1}\left(a_{j, 0} \cdot e+\partial\left(\left(a_{j, 0}\right)_{0} e\right)\right. \\
& =0, \text { and } \\
& =e(-1) b(2) e+\left(b_{0} e\right)(1) e+2\left(b_{1} e\right)(0) e \\
b(2) e(-1) e & =\left(\beta_{f}(-h)+\beta_{h}(2 e)\right)_{1} e+2\left(\beta_{f} \mathbf{1}+\sum_{j=1}^{l} \beta_{j, 1} a_{j, 0}\right)_{0} e \\
& =0 .
\end{aligned}
$$

Hence, $(b(-1) \mathbf{1})_{n} e(-1) e=b(n) e(-1) e=0$ for all $n \geq 1$. Let $m \geq 2$. For $t \geq 1$,

$$
\begin{aligned}
& (b(-m) \mathbf{1})_{t} e(-1) e \\
& =\sum_{i \geq 0}(-1)^{i}\left(\begin{array}{c}
-m \\
i
\end{array}\right)\left(b(-m-i) \mathbf{1}(t+i)-(-1)^{-m} \mathbf{1}(-m+t-i) b(i)\right) e(-1) e \\
& =-(-1)^{-m} \mathbf{1}(-m+t) b(0) e(-1) e .
\end{aligned}
$$

This implies that $(b(-m) \mathbf{1})_{m+1} e(-1) e=0$ and $(b(-m) \mathbf{1})_{m} e(-1) e=0$.

Recall that for $n \geq 1$,

$$
\begin{aligned}
& \left(V_{B}\right)_{(n)} \\
& =\operatorname{span}\left\{b_{1}\left(-n_{1}\right) \ldots . . b_{k}\left(-n_{k}\right) \mathbf{1} \mid b_{i} \in B, n_{1} \geq \ldots \geq n_{k} \geq 1, n_{1}+\ldots+n_{k}=n\right\} .
\end{aligned}
$$


If $v \in V_{B}$ is of the form $b_{1}\left(-n_{1}\right) \ldots . . b_{k}\left(-n_{k}\right) \mathbf{1}$ where $b_{i} \in\left\{e, f, h, \partial\left(a_{j, i}\right) \mid j \in\{1, \ldots, l\}, i \in\right.$ $\{0,1\}\}$, we say that $v$ is a monomial vector that has length $k$. For the third step, we will show that if $b \in B$, and $v$ is a monomial vector of length $k$ then either $b(0) v=0$ or $b(0) v$ is a sum of monomial vectors that have length $k$. Clearly, if $b \in \partial(A)$ then $b_{0} v=0$. Notice that

$$
\begin{aligned}
& b(0) b^{1}\left(-n_{1}\right) \mathbf{1}=\left(b_{0} b^{1}\right)\left(-n_{1}\right) \mathbf{1} \\
& b(0) b^{1}\left(-n_{1}\right) b^{2}\left(-n_{2}\right) \mathbf{1}=b^{1}\left(-n_{1}\right)\left(b_{0} b^{2}\right)\left(-n_{2}\right) \mathbf{1}+\left(b_{0} b^{1}\right)\left(-n_{1}\right) b^{2}\left(-n_{2}\right) \mathbf{1}
\end{aligned}
$$

If $b_{0} b^{1}=0$ then $b(0) b^{1}\left(-n_{1}\right) \mathbf{1}=0$. If $b_{0} b^{1} \neq 0$ then $b(0) b^{1}\left(-n_{1}\right) \mathbf{1}$ is a sum of monomial vectors of length 1 . Similarly, if $b_{0} b^{2}=0=b_{0} b^{1}$ then $b(0) b^{1}\left(-n_{1}\right) b^{2}\left(-n_{2}\right) \mathbf{1}=0$. Otherwise, $b(0) b^{1}\left(-n_{1}\right) b^{2}\left(-n_{2}\right) \mathbf{1}$ is a sum of monomial vectors of length 2 . Now, we assume that for monomial vectors $w$ of length $t$, either $b(0) w=0$ or $b(0) w$ are the sum of monomial vectors of length $t$. Since

$$
\begin{aligned}
& b(0) b_{1}\left(-n_{1}\right) \ldots . . b_{t+1}\left(-n_{t+1}\right) \mathbf{1} \\
& =b_{1}\left(-n_{1}\right) b(0) b_{2}\left(-n_{2}\right) \ldots . . b_{t+1}\left(-n_{t+1}\right) \mathbf{1} \\
& \quad+\left(b_{0} b^{1}\right)\left(-n_{1}\right) b_{2}\left(-n_{2}\right) \ldots . . b_{t+1}\left(-n_{t+1}\right) \mathbf{1},
\end{aligned}
$$

by induction hypothesis, we can conclude that either $b(0) b_{1}\left(-n_{1}\right) \ldots . . b_{t+1}\left(-n_{t+1}\right) \mathbf{1}=0$ or $b(0) b_{1}\left(-n_{1}\right) \ldots . . b_{t+1}\left(-n_{t+1}\right) \mathbf{1}$ is a sum of monomial vectors of length $t+1$.

For the fourth step, we will show that for every monomial vector $v \in V_{B}, v_{(\operatorname{deg} v)+1} e(-1) e=0$ and $v_{\operatorname{deg} v} e(-1) e=0$. We will use an induction on the length of monomial vectors to prove this statement. By the first step and the second step, we can conclude immediately that if $v$ is a monomial vector of length $q$ where $0 \leq q \leq 1$ then $v_{(\operatorname{deg} v)+1} e(-1) e=0$ and $v_{(\operatorname{deg} v)} e(-1) e=0$. Now, we assume that for any monomial vector $v$ of length $k \leq t, v_{(\operatorname{deg} v)+1} e(-1) e=0$ and $v_{(\operatorname{deg} v)} e(-1) e=0$. For $i \in\{1, \ldots, t+1\}$, we let $n_{i}$ be a positive integer, $b^{i} \in B$. We set $n=n_{1}+n_{2}+\ldots+n_{t}+n_{t+1}+1$. Notice that

$$
\begin{aligned}
& \left(b^{1}\left(-n_{1}\right) b^{2}\left(-n_{2}\right) \ldots b^{t}\left(-n_{t}\right) b^{t+1}\left(-n_{t+1}\right) \mathbf{1}\right)_{n} e(-1) e \\
= & \sum_{i \geq 0}(-1)^{i}\left(\begin{array}{c}
-n_{1} \\
i
\end{array}\right)\left(b^{1}\left(-n_{1}-i\right)\left(b^{2}\left(-n_{2}\right) \ldots b^{t}\left(-n_{t}\right) b^{t+1}\left(-n_{t+1}\right) \mathbf{1}\right)_{n+i}\right. \\
& \left.\quad-(-1)^{n_{1}}\left(b^{2}\left(-n_{2}\right) \ldots b^{t}\left(-n_{t}\right) b^{t+1}\left(-n_{t+1}\right) \mathbf{1}\right)_{-n_{1}+n-i} b^{1}(i)\right) e(-1) e \\
= & -(-1)^{n_{1}}\left(b^{2}\left(-n_{2}\right) \ldots b^{t+1}\left(-n_{t+1}\right) \mathbf{1}\right)_{-n_{1}+n} b^{1}(0) e(-1) e \quad \text { (by induction hypothesis) } \\
= & (-1)^{n_{1}+1}\left(b^{1}(0)\left(b^{2}\left(-n_{2}\right) \ldots b^{t+1}\left(-n_{t+1}\right) \mathbf{1}\right)_{-n_{1}+n} e(-1) e\right. \\
& \left.\quad-\left(b^{1}(0) b^{2}\left(-n_{2}\right) \ldots b^{t+1}\left(-n_{t+1}\right) \mathbf{1}\right)_{-n_{1}+n} e(-1) e\right),
\end{aligned}
$$

For simplicity, we set

$$
u=b^{2}\left(-n_{2}\right) \ldots b^{t+1}\left(-n_{t+1}\right) \mathbf{1} \text { and } w=b^{1}(0) b^{2}\left(-n_{2}\right) \ldots b^{t+1}\left(-n_{t+1}\right) \mathbf{1} .
$$

Note that the vector $u$ is a monomial vector of length $t$, the vector $w$ is a sum of monomial vectors of length $t$, and

$$
-n_{1}+n=n_{2}+\ldots .+n_{t+1}+1=(\operatorname{deg} u)+1=(\operatorname{deg} w)+1 .
$$


By induction hypothesis, we can conclude that

$$
\left(b^{1}\left(-n_{1}\right) b^{2}\left(-n_{2}\right) \ldots b^{t}\left(-n_{t}\right) b^{t+1}\left(-n_{t+1}\right) \mathbf{1}\right)_{n} e(-1) e=0 .
$$

Next, we assume that $n=n_{1}+n_{2}+\ldots+n_{t+1}$. We have

$$
\begin{aligned}
& \left(b^{1}\left(-n_{1}\right) b^{2}\left(-n_{2}\right) \ldots b^{t+1}\left(-n_{t+1}\right) \mathbf{1}\right)_{n} e(-1) e \\
& =\sum_{i \geq 0}(-1)^{i}\left(\begin{array}{c}
-n_{1} \\
i
\end{array}\right)\left(b^{1}\left(-n_{1}-i\right)\left(b^{2}\left(-n_{2}\right) \ldots b^{t+1}\left(-n_{t+1}\right) \mathbf{1}\right)_{n+i}\right. \\
& \left.\quad-(-1)^{n_{1}}\left(b^{2}\left(-n_{2}\right) \ldots b^{t+1}\left(-n_{t+1}\right) \mathbf{1}\right)_{-n_{1}+n-i} b^{1}(i)\right) e(-1) e \\
& =\delta_{n_{1}, 1} b^{1}\left(-n_{1}\right)\left(b^{2}\left(-n_{2}\right) \ldots b^{t+1}\left(-n_{t+1}\right) \mathbf{1}\right)_{n} e(-1) e \\
& \quad-(-1)^{n_{1}}\left(b^{2}\left(-n_{2}\right) \ldots b^{t+1}\left(-n_{t+1}\right) \mathbf{1}\right)_{-n_{1}+n} b^{1}(0) e(-1) e \\
& =\delta_{n_{1}, 1} b^{1}\left(-n_{1}\right)\left(b^{2}\left(-n_{2}\right) \ldots b^{t+1}\left(-n_{t+1}\right) \mathbf{1}\right)_{n} e(-1) e \\
& \quad-(-1)^{n_{1}}\left\{b^{1}(0)\left(b^{2}\left(-n_{2}\right) \ldots b^{t+1}\left(-n_{t+1}\right) \mathbf{1}\right)_{-n_{1}+n}\right. \\
& \left.\quad-\left(b^{1}(0) b^{2}\left(-n_{2}\right) \ldots b^{t+1}\left(-n_{t+1}\right) \mathbf{1}\right)_{-n_{1}+n}\right\} e(-1) e .
\end{aligned}
$$

We set $p=b^{2}\left(-n_{2}\right) \ldots b^{t+1}\left(-n_{t+1}\right) \mathbf{1}$ and $r=b^{1}(0) b^{2}\left(-n_{2}\right) \ldots b^{t+1}\left(-n_{t+1}\right) \mathbf{1}$. Notice that when $n_{1}=1, p_{n}=p_{(\operatorname{deg} p)+1}$. Also, $p_{-n_{1}+n}=p_{\operatorname{deg} p}, r$ is a sum of monomial vectors of length $t$ and $r_{-n_{1}+n}=r_{\operatorname{deg} r}$. By induction hypothesis, we can conclude that

$$
\left(b^{1}\left(-n_{1}\right) b^{2}\left(-n_{2}\right) \ldots b^{t+1}\left(-n_{t+1}\right) \mathbf{1}\right)_{n} e(-1) e=0 .
$$

Hence, for any homogeneous monomial vector $v$ of length $k, v_{(\operatorname{deg} v)+1} e(-1) e=0$ and $v_{\operatorname{deg} v} e(-1) e=$ 0 . This completes the fourth step.

Because $v_{(\operatorname{deg} v)+1} e(-1) e=0$ and $v_{\operatorname{deg} v} e(-1) e=0$ for any homogeneous monomial vector $v$ of any length $k$, we can conclude further that for any homogeneous vector $u \in V_{B}$,

$$
\begin{aligned}
& u_{(\operatorname{deg} u)+1} e(-1) e=0, \text { and } \\
& u_{\operatorname{deg} u} e(-1) e=0 .
\end{aligned}
$$

Moreover, for any homogeneous vector $v \in V_{B}, t \in \mathbb{Z}$, we have $v_{t} e(-1) e \in \oplus_{n=2}^{\infty}\left(V_{B}\right)_{(n)}$. This implies that for $v \in V_{B}, t \in \mathbb{Z}$, we have $v_{t} e(-1) e \in \oplus_{n=2}^{\infty}\left(V_{B}\right)_{(n)}$.

Next, we will show that for $i \geq 1, v_{t} D^{i} e(-1) e \in \oplus_{n=2}^{\infty}\left(V_{B}\right)_{(n)}$ for all $v \in V_{B}, t \in \mathbb{Z}$. Clearly,

$$
v_{t} D e(-1) e=D v_{t} e(-1) e+t v_{t-1} e(-1) e \in \oplus_{n=2}^{\infty}\left(V_{B}\right)_{(n)} .
$$

Now, let us assume that $v_{t} D^{j} e(-1) e \in \oplus_{n=2}^{\infty}\left(V_{B}\right)_{(n)}$ for all $v \in V_{B}, t \in \mathbb{Z}$. Since

$$
v_{t} D^{j+1} e(-1) e=D v_{t} D^{j} e(-1) e+t v_{t-1} D^{j} e(-1) e,
$$

we can conclude immediately that $v_{t} D^{j+1} e(-1) e \in \oplus_{n=2}^{\infty}\left(V_{B}\right)_{(n)}$. Hence,

$$
v_{t} D^{i} e(-1) e \in \oplus_{n=2}^{\infty}\left(V_{B}\right)_{(n)} \text { for all } i \geq 0, v \in V_{B}, t \in \mathbb{Z} .
$$

This implies that $(e(-1) e) \cap(A \oplus B)=\{0\}$.

We set

$$
\overline{V_{B}}=V_{B} /(e(-1) e)
$$


Proposition 37. $\overline{V_{B}}=\oplus_{n=0}^{\infty}\left(\overline{V_{B}}\right)_{(n)}$ is an indecomposable non-simple $\mathbb{N}$-graded vertex algebra such that $\left(\overline{V_{B}}\right)_{(0)}=A$ and $\left(\overline{V_{B}}\right)_{(1)}=B$.

Proof. Since $\overline{V_{B}}=\oplus_{n=0}^{\infty}\left(\overline{V_{B}}\right)_{(n)}$ is a $\mathbb{N}$-graded vertex algebra such that $\left(\overline{V_{B}}\right)_{(0)}=A$ and $\left(\overline{V_{B}}\right)_{(1)}=B$, by Proposition 19 , we can conclude that $\overline{V_{B}}=\oplus_{n=0}^{\infty}\left(\overline{V_{B}}\right)_{(n)}$ is an indecomposable non-simple $\mathbb{N}$-graded vertex algebra.

This completes the proof of statement $(i)$ of Theorem 2 .

To prove statement $(i i)$ and statement (iii) of Theorem 2, we need to use properties of vertex operator algebras associated with a certain type of rank one lattices, and vertex operator algebras associated with highest weight representations of affince Lie algebras. We provide background material on these topics in Appendices.

Let $\hat{S}=S \otimes \mathbb{C}\left[t, t^{-1}\right] \oplus \mathbb{C} c$ be the affine Lie algebra where $c$ is central and

$$
\left[u \otimes t^{m}, v \otimes^{n}\right]=[u, v] \otimes t^{m+n}+m\langle\langle u, v\rangle\rangle \delta_{m+n, 0} c .
$$

Here, $\langle\langle\rangle$,$\rangle is a symmetric invariant bilinear form of S$ such that $\langle\langle e, f\rangle\rangle=1,\langle\langle h, h\rangle\rangle=2$ and $\langle\langle e, e\rangle\rangle=\langle\langle f, f\rangle\rangle=\langle\langle e, h\rangle\rangle=\langle\langle f, h\rangle\rangle=0$. The generalized Verma $\hat{S}$-module $M_{S}(k, 0)$ is a vertex operator algebra (see Appendices for the construction of the vertex operator algebra $M_{S}(k, 0)$ and its properties).

For $u \in \overline{V_{B}}$, we set $Y_{\overline{V_{B}}}(u, z)=\sum_{n \in \mathbb{Z}} u[n] z^{-n-1}$. Since $S=\operatorname{Span}\{e, f, h\}$ is a subset of $\left(\overline{V_{B}}\right)_{(1)}$ and $S$ is a Lie algebra with a symmetric invariant bilinear form $\langle\rangle:, S \times S \rightarrow \mathbb{C}$ such that $\left\langle s, s^{\prime}\right\rangle \mathfrak{e}=s[1] s^{\prime}$, the map $\hat{S} \rightarrow \operatorname{End}\left(\overline{V_{B}}\right): s \otimes t^{m} \rightarrow s[m]$ is a representation of the affine KacMoody algebra $\hat{S}$ of level $k$ where $\left\langle s, s^{\prime}\right\rangle=k\left\langle\left\langle s, s^{\prime}\right\rangle\right\rangle$ for $s, s^{\prime} \in S$. Since $\langle h, h\rangle_{\mathfrak{e}}=h[1] h=2 \mathfrak{e}$ and $\langle\langle h, h\rangle\rangle=2$, we then have that $k=1$. Moreover, $\overline{V_{B}}$ is a module of $M_{S}(1,0)$.

Let $U$ be the vertex sub-algebra of $\overline{V_{B}}$ that is generated by $S$. This vertex algebra $U$ is a highest weight module for $\hat{S}$. In fact, $U$ is a quotient of the generalized Verma module $M_{S}(1,0)$. Notice that $U$ is integrable if and only if $U \cong L(1,0)$. By Theorem 10.7 in $[\mathrm{K}$, this is equivalent to the condition $(e[-1])^{l} \mathbf{1}=0$ for some $l \geq 0$. Since $(e[-1])^{2} \mathbf{1}=0$, we can conclude immediately that $U$ is integrable. Indeed, $U$ is isomorphic to $L(1,0)$. Moreover, by Proposition 25, and Theorem 13.16 of [DoL], $\overline{V_{B}}$ is integrable as $\hat{S}$-module.

By Proposition 47, we have $f[-1] f=0$.

\section{Lemma 38.}

(i) $((f+h-e)[-1])^{2} \mathbf{1}=0$.

(ii) If $\left(W, Y_{W}\right)$ is a $\overline{V_{B}}$-module then $Y_{W}(e, z)^{2}=Y_{W}(f, z)^{2}=Y_{W}(f+h-e, z)^{2}=0$.

(iii) In particular, we have $Y_{\overline{V_{B}}}(e, z)^{2}=Y_{\overline{V_{B}}}(f, z)^{2}=Y_{\overline{V_{B}}}(f+h-e, z)^{2}=0$ on $\overline{V_{B}}$.

Proof. Since

$$
\begin{aligned}
& 0=e[0](f[-1])^{2} \mathbf{1}=f[-1] h[-1] \mathbf{1}+h[-1] f[-1] \mathbf{1} \\
& 0=f[0](e[-1])^{2} \mathbf{1}=-(e[-1] h[-1] \mathbf{1}+h[-1] e[-1] \mathbf{1}) \text { and } \\
& 0=(f[0])^{2}(e[-1])^{2} \mathbf{1}=-\left(2 e[-1] f[-1] \mathbf{1}-2(h[-1])^{2} \mathbf{1}+2 f[-1] e[-1] \mathbf{1}\right)
\end{aligned}
$$


we can conclude that $((f+h-e)[-1])^{2} \mathbf{1}=0$. This proves $(i)$.

By Proposition 25, we can conclude that if $\left(W, Y_{W}\right)$ is a $\overline{V_{B}}$-module then $Y_{W}(e, z)^{2}=$ $Y_{W}(f, z)^{2}=Y_{W}(f+h-e, z)^{2}=0$. In particular, we have $Y_{\overline{V_{B}}}(e, z)^{2}=Y_{\overline{V_{B}}}(f, z)^{2}=$ $Y_{\overline{V_{B}}}(f+h-e, z)^{2}=0$ on $\overline{V_{B}}$. We obtain statments $(i i)$ and $(i i i)$ as desired.

Lemma 39. $\overline{V_{B}}$ satisfies the $C_{2}$-condition.

Proof. Clearly,

$\overline{V_{B}} / C_{2}\left(\overline{V_{B}}\right)=\operatorname{Span}\left\{a+C_{2}\left(\overline{V_{B}}\right), b+C_{2}\left(\overline{V_{B}}\right), b^{1}[-1] \ldots b^{k}[-1] \mathbf{1}+C_{2}\left(\overline{V_{B}}\right) \mid a \in A, b, b^{i} \in S, k \geq 2\right\}$.

Now, we follow the proof of Proposition 12.6 in DoLiM1. Since $\{e, f, h\}$ forms a basis of $S$, this implies that $\{e, f, f+h-e\}$ forms a basis for $S$ as well. Observe that for $u, v \in$ $\{e, f, f+h-e\}, w \in \overline{V_{B}}, u[-1] v[-1] w=v[-1] u[-1] w+\left(u_{0} v\right)[-2] w$. Since

$$
e[-1] e=f[-1] f=(f+h-e)[-1](f+h-e)=0,
$$

we can conclude that

$$
\begin{aligned}
\overline{V_{B}} / C_{2}\left(\overline{V_{B}}\right)=\operatorname{Span}\left\{a+C_{2}\left(\overline{V_{B}}\right), b+C_{2}\left(\overline{V_{B}}\right), u[-1] v+C_{2}\left(\overline{V_{B}}\right),\right. \\
\left.u[-1] v[-1] w+C_{2}\left(\overline{V_{B}}\right) \mid a \in A, b, u, v, w \in\{e, f, f+h-e\}\right\},
\end{aligned}
$$

and $\overline{V_{B}}$ is $C_{2}$-cofinite.

This completes the proof of statement $(i i)$ of Theorem 2

Next, we will study $\mathbb{N}$-graded $\overline{V_{B}}$-modules. Observe that $A \oplus B$ generates $\overline{V_{B}}$ as a vertex algebra. Consequently, if $W$ is a $\overline{V_{B}}$-module, then $W$ is a restricted $\mathcal{L}$-module with $u(n)$ acting as $u_{n}$ for $u \in A \oplus B, n \in \mathbb{Z}$. Moreover, the set of $\overline{V_{B}}$-submodules is the set of $\mathcal{L}$-submodules.

Proposition 40. [LiY1] Let $\left(W, Y_{W}\right)$ be a $V_{\mathcal{L}}$-module. Assume that for any $a, a^{\prime} \in A, b \in B$,

$$
\begin{aligned}
& Y_{W}(\mathfrak{e}, z) u=u, \\
& Y_{W}\left(a(-1) a^{\prime}, z\right) u=Y_{W}\left(a * a^{\prime}, z\right) u, \\
& Y_{W}(a(-1) b, z) u=Y_{W}(a \cdot b, z) u,
\end{aligned}
$$

for all $u \in U$ where $U$ is a generating subspace of $W$ as a $V_{\mathcal{L}}$-module, then $W$ is naturally a $\overline{V_{B}}$-module.

Lemma 41. Let $\left(Q, Y_{Q}\right)$ be a $V_{B}$-module such that $Y(e(-1) e, z) u=0$ for all $u \in F$ where $F$ is a generating subspace of $Q$ as a $V_{B}$-module. Then $Q$ is a $\overline{V_{B}}$-module.

Proof. By using Proposition 26, one can obtain the above statement very easily.

Lemma 42. Let $W=\oplus_{n=0}^{\infty} W_{(n)}$ be a $\mathbb{N}$-graded $\overline{V_{B}}$-module with $W_{(0)} \neq\{0\}$. Then

(i) $W_{(0)}$ is an A-module with $a \cdot w=a_{-1} w$ for $a \in A, w \in W_{(0)}$, and $W_{(0)}$ is a module for the Lie algebra $B / A \partial(A)\left(\cong l_{2}\right)$ with $b \cdot w=b_{0} w$ for $b \in B, w \in W_{(0)}$. Furthermore, $W_{(0)}$ equipped with these module structures is a module for the Lie A-algebroid $B / A \partial(A)$

(ii) Moreover, $e_{0}\left(e_{0} w\right)=0, e_{-1}\left(e_{-1} w\right)=0, f_{0}\left(f_{0} w\right)=0$ and $f_{-1}\left(f_{-1} w\right)=0$ for all $w \in W_{(0)}$. 
(iii) If $W$ is simple then $W_{(0)}$ is an irreducible module for Lie A-algebroid $B / A \partial(A)$ that has dimension either 1 or 2. Moreover, for $j \in\{1, \ldots, l\}, i \in\{0,1\}, a_{j, i}$ acts trivially on $W_{(0)}$.

Proof. By following the proof of Proposition 4.8 of [LiY1], one can shows that statements (i) and the statement "if $W$ is simple then $W_{(0)}$ is a simple $B / A \partial(A)$-module" hold.

Now, we will prove that $e_{0} e_{0} w=f_{0} f_{0} w=0$ for all $w \in W_{(0)}$. By Proposition 38, we have $Y_{W}(e, z)^{2}=0$ and $Y_{W}(f, z)^{2}=0$ on $W$. Since $e_{n} u=0$ for all $n \geq 1, u \in W_{(0)}$, these imply that $Y(e, z)^{2} u=\sum_{n \geq 0} \sum_{m \geq 0} e_{-m} e_{-n} u z^{m+n-2}$. Moreover, the coefficient of $z^{-2}$ is $e_{0} e_{0} u=0$ and the constant term is $e_{-1} e_{-1} u=0$. Similarly, using the fact that $Y_{W}(f, z)^{2}=0$ and $f_{n} u=0$ for all $n \geq 1, u \in W_{(0)}$, one can show that

$$
f_{0} f_{0} u=0 \text { and } f_{-1} f_{-1} u=0 .
$$

We obtain statement (ii) as desired.

Next, we prove statement (iii). We only need to show that for an irreducible $\overline{V_{B}}$-module $W$, $W_{(0)}$ is either one dimensional or two dimensional. By statement (ii), $W_{(0)}$ has either one dimensional or two dimensional. Recall that $\left\{\mathfrak{e}, a_{j, i} \mid j \in\{1, \ldots, l\}, i \in\{0,1\}\right\}$ is a basis of $A$, $B / A \partial(A) \cong s l_{2}$, and

$$
v_{0}\left(a_{-1} w\right)-a_{-1}\left(v_{0} w\right)=\left(v_{0} a\right)_{-1} w \text { for all } a \in A, v \in B / A \partial A, w \in W_{(0)},
$$

and $e_{0}\left(a_{j, 1}\right)=a_{j, 0}, f_{0}\left(a_{j, 0}\right)=a_{j, 1}$.

If $\operatorname{dim} W_{(0)}=1$ then $W_{(0)}$ is a trivial module of $s l_{2}$. For simplicity, we set $W=\mathbb{C} w_{0}$. By equation (22), we have

$$
\begin{aligned}
& 0=e_{0}\left(\left(a_{j, 1}\right)_{-1} w_{0}\right)-\left(a_{j, 1}\right)_{-1} e_{0} w_{0}=\left(e_{0}\left(a_{j, 1}\right)\right)_{-1} w_{0}=\left(a_{j, 0}\right)_{-1} w_{0}, \text { and } \\
& 0=f_{0}\left(\left(a_{j, 0}\right)_{-1} w_{0}\right)-\left(a_{j, 0}\right)_{-1} f_{0} w_{0}=\left(f_{0}\left(a_{j, 0}\right)\right)_{-1} w_{0}=\left(a_{j, 1}\right)_{-1} w_{0} .
\end{aligned}
$$

We now assume that $\operatorname{dim} W_{(0)}=2$. Then $W_{(0)}=\operatorname{Span}\left\{w_{0}, w_{1}\right\}$ where $w_{0}$ is the highest weight vector of $W_{(0)}$ of weight 1 and $w_{1}=f_{0} w_{0}$. Recall that for $a \in A, b \in B / A \partial(A)$, $a_{-1}\left(b_{0} w\right)=\left(a_{-1} b\right)_{0} w$ for all $w \in W_{(0)}$. Hence, we have

$$
\begin{aligned}
& \left(a_{j, 0}\right)_{-1} w_{0}=\left(a_{j, 0}\right)_{-1}\left(e_{0} w_{1}\right)=\left(\left(a_{j, 0}\right)_{-1} e\right)_{0} w_{1}=0, \\
& \left(a_{j, 0}\right)_{-1} w_{1}=\left(a_{j, 0}\right)_{-1}\left(f_{0} w_{0}\right)=\left(\left(a_{j, 0}\right)_{-1} f\right)_{0} w_{0}=\left(\partial\left(a_{j, 1}\right)\right)_{0} w_{0}=0, \\
& \left(a_{j, 1}\right)_{-1} w_{0}=\left(a_{j, 1}\right)_{-1}\left(e_{0} w_{1}\right)=\left(\left(a_{j, 1}\right)_{-1} e\right)_{0} w_{1}=\left(\partial\left(a_{j, 0}\right)\right)_{0} w_{1}=0, \\
& \left(a_{j, 1}\right)_{-1} w_{1}=\left(a_{j, 1}\right)_{-1}\left(f_{0} w_{0}\right)=\left(\left(a_{j, 1}\right)_{-1} f\right)_{0} w_{0}=0 .
\end{aligned}
$$

This completes the proof of statement (iii).

Lemma 43. Let $W$ be a $\mathbb{N}$-graded $\overline{V_{B}}$-module with $W_{(0)} \neq\{0\}$. If $W$ is an irreducible module for the vertex algebra $L(1,0)$, then $W$ is an irreducible $\overline{V_{B}}$-module.

Proof. Let $W$ be a $\mathbb{N}$-graded $\overline{V_{B}}$-module with $W_{(0)} \neq\{0\}$. Assume that $W$ is an irreducible module for the vertex algebra $L(1,0)$. Let $G \neq\{0\}$ be $\overline{V_{B}}$-submodule of $W$. Hence, $G$ is a $U$-submodule of $W$. Since $U$ is isomorphic to $L(1,0)$ as vertex algebra, we can conclude that $G=W$. Consequently, $W$ is an irreducible $\overline{V_{B}}$-module. 
Lemma 44. Let $L=\mathbb{Z} \alpha$ be a positive definite even lattice of rank one equipped with a $\mathbb{Q}$-valued $\mathbb{Z}$-bilinear form $(\cdot, \cdot)$ such that $(\alpha, \alpha)=2$. Then the vertex operator algebra $V_{L}=\oplus_{n=0}^{\infty}\left(V_{L}\right)_{(n)}$ is an irreducible $\mathbb{N}$-graded $\overline{V_{B}}$-module such that $\operatorname{dim}\left(V_{L}\right)_{(0)}=1$ and $V_{L+\frac{1}{2} \alpha}=\oplus_{n=0}^{\infty}\left(V_{L+\frac{1}{2} \alpha}\right)_{(n)}$ is an irreducible $\mathbb{N}$-graded $\overline{V_{B}}$-module such that $\operatorname{dim}\left(V_{L+\frac{1}{2} \alpha}\right)_{(0)}=2$.

Proof. Recall that $\left(V_{L}, Y\right)$ and $\left(V_{L+\frac{1}{2} \alpha}, Y\right)$ are irreducible modules of $L(1,0)$ (see Appendices). By Lemma 43, to show that $V_{L}$ and $V_{L+\frac{1}{2} \alpha}$ are irreducible $\overline{V_{B}}$-modules, we only need to show that they are actually $\overline{V_{B}}$-modules. Let $L^{\circ}$ be the dual lattice of $L$. To prove that $V_{L}$ and $V_{L+\frac{1}{2} \alpha}$ are irreducible $\overline{V_{B}}$-modules, we only need to show that $V_{L^{\circ}}=V_{L} \oplus V_{L+\frac{1}{2} \alpha}$ is a $\overline{V_{B^{-}}}$ module.

Recall that for $\gamma \in \mathbb{H}, \beta \in L$,

$$
\begin{aligned}
& Y(\gamma, z)=Y(\gamma(-1) \mathbf{1}, z)=\sum_{n \in \mathbb{Z}} \gamma(n) z^{-n-1}, \\
& Y\left(e^{\beta}, z\right)=\sum_{n \in \mathbb{Z}}\left(e^{\beta}\right)_{n} z^{-n-1}=\exp \left(\sum_{m=1}^{\infty} \beta(-m) \frac{z^{m}}{m}\right) \exp \left(-\sum_{m=1}^{\infty} \beta(m) \frac{z^{-m}}{m}\right) e^{\beta} z^{\beta} .
\end{aligned}
$$

For $n \in \mathbb{Z}$, we assume that $h \otimes t^{n}$ acts as $\alpha(n), e \otimes t^{n}$ acts as $\left(e^{\alpha}\right)_{n}, f \otimes t^{n}$ acts as $\left(e^{-\alpha}\right)_{n}$, $a_{j, i} \otimes t^{n}$ acts as zero, $\partial\left(a_{j, i}\right) \otimes t^{n}$ acts as zero and $\mathfrak{e} \otimes t^{n}$ acts as $\delta_{n,-1} 1$. First we claim that $\left(V_{L^{\circ}}, Y_{V_{L^{\circ}}}\right)$ is a $V_{\mathcal{L}^{-}}$-module. Let $a=\lambda_{\mathfrak{e}} \mathfrak{e}+\sum_{j=1}^{l} \sum_{i=0}^{1} \lambda_{j, i} a_{j, i}$. Notice that

$$
Y_{V_{L^{\circ}}}(a, z)=\lambda_{\mathfrak{e}} Y_{V_{L^{\circ}}}(\mathfrak{e}, z)=\lambda_{\mathfrak{e}} I d_{V_{L^{\circ}}} .
$$

Here, $I d_{V_{L^{\circ}}}$ is the identity map on $V_{L^{\circ}}$. The following are commutator relations among $\alpha$, $e^{ \pm \alpha}$ on $V_{L^{\circ}}$ :

$$
\begin{aligned}
{\left[Y\left(\alpha, z_{1}\right), Y\left(\alpha, z_{2}\right)\right] } & =-2 \frac{\partial}{\partial z_{1}} z_{2}^{-1} \delta\left(\frac{z_{1}}{z_{2}}\right)=2 \frac{\partial}{\partial z_{2}} z_{2}^{-1} \delta\left(\frac{z_{1}}{z_{2}}\right), \\
{\left[Y\left(\alpha, z_{1}\right), Y\left(e^{ \pm \alpha}, z_{2}\right)\right] } & = \pm 2 z_{2}^{-1} \delta\left(\frac{z_{1}}{z_{2}}\right) Y\left(e^{ \pm \alpha}, z_{2}\right) \\
{\left[Y\left(e^{\alpha}, z_{1}\right), Y\left(e^{-\alpha}, z_{2}\right)\right] } & =z_{2}^{-1} \delta\left(\frac{z_{1}}{z_{2}}\right) Y\left(\alpha, z_{2}\right)-\frac{\partial}{\partial z_{1}} z_{2}^{-1} \delta\left(\frac{z_{1}}{z_{2}}\right), \\
& =z_{2}^{-1} \delta\left(\frac{z_{1}}{z_{2}}\right) Y\left(\alpha, z_{2}\right)+\frac{\partial}{\partial z_{2}} z_{2}^{-1} \delta\left(\frac{z_{1}}{z_{2}}\right), \\
{\left[Y\left(e^{\alpha}, z_{1}\right), Y\left(e^{\alpha}, z_{2}\right)\right] } & =\left[Y\left(e^{-\alpha}, z_{1}\right), Y\left(e^{-\alpha}, z_{2}\right)\right]=0 .
\end{aligned}
$$

By comparing these commutator relations with commutator relations (19)-(21), we can conclude that $V_{L^{\circ}}$ is a $\mathcal{L}$-module. Hence, $V_{L^{\circ}}$ is a $V_{\mathcal{L}^{-} \text {-module. }}$

Let $a=\lambda_{\mathfrak{e}} \mathfrak{e}+\sum_{j=1}^{l} \sum_{i=0}^{1} \lambda_{j, i} a_{j, i}, a^{\prime}=\lambda_{\mathfrak{e}}^{\prime} \mathfrak{e}+\sum_{j=1}^{l} \sum_{i=0}^{1} \lambda_{j, i}^{\prime} a_{j, i} \in A$. Since

$$
Y_{V_{L^{\circ}}}\left(a * a^{\prime}, z_{2}\right)=\lambda_{\mathfrak{e}} \lambda_{\mathfrak{e}}^{\prime} I d_{V_{L^{\circ}}}
$$


and

$$
\begin{aligned}
Y_{V_{L^{\circ}}}\left(a(-1) a^{\prime}, z_{2}\right)= & \operatorname{Res}_{z_{0}}\left\{z _ { 0 } ^ { - 1 } \operatorname { R e s } _ { z _ { 1 } } \left\{z_{0}^{-1} \delta\left(\frac{z_{1}-z_{2}}{z_{0}}\right) Y_{V_{L^{\circ}}}\left(a, z_{1}\right) Y_{V_{L^{\circ}}}\left(a^{\prime}, z_{2}\right)\right.\right. \\
& \left.\left.\quad-z_{0}^{-1} \delta\left(\frac{z_{2}-z_{1}}{-z_{0}}\right) Y_{V_{L^{\circ}}}\left(a^{\prime}, z_{2}\right) Y_{V_{L^{\circ}}}\left(a, z_{1}\right)\right\}\right\} \\
= & \operatorname{Res}_{z_{0}} z_{0}^{-1} \operatorname{Res}_{z_{1}} z_{2}^{-1} \delta\left(\frac{z_{1}-z_{0}}{z_{2}}\right) \lambda_{\mathfrak{e}} \lambda_{\mathfrak{e}}^{\prime} I d_{V_{L^{\circ}}} \\
= & \lambda_{\mathfrak{e}} \lambda_{\mathfrak{e}}^{\prime} I d_{V_{L^{\circ}}},
\end{aligned}
$$

we can conclude that

$$
Y_{V_{L^{\circ}}}\left(a(-1) a^{\prime}, z_{2}\right)=Y_{V_{L^{\circ}}}\left(a * a^{\prime}, z_{2}\right)
$$

Let $b=\rho_{e} e+\rho_{f} f+\rho_{h} h+\sum_{j=1}^{l} \sum_{i=0}^{1} \rho_{j, i} \partial\left(a_{j, i}\right)$. Notice that

$$
Y_{V_{L^{\circ}}}(b, z)=\rho_{e} Y_{V_{L^{\circ}}}(e, z)+\rho_{f} Y_{V_{L^{\circ}}}(f, z)+\rho_{h} Y_{V_{L^{\circ}}}(h, z) .
$$

Since

$$
\begin{aligned}
a \cdot b \quad & =\left(\lambda_{\mathfrak{e}} \mathfrak{e}+\sum_{j=1}^{l} \sum_{i=0}^{1} \lambda_{j, i} a_{j, i}\right) \cdot\left(\rho_{e} e+\rho_{f} f+\rho_{h} h+\sum_{j=1}^{l} \sum_{i=0}^{1} \rho_{j, i} \partial\left(a_{j, i}\right)\right. \\
& =\lambda_{\mathfrak{e}} b+\tau \text { where } \tau \in \partial(A)
\end{aligned}
$$

we then have that $Y_{V_{L^{\circ}}}(a \cdot b, z)=Y_{V_{L^{\circ}}}\left(\lambda_{\mathfrak{e}} b, z\right)=\lambda_{\mathfrak{e}} Y_{V_{L^{\circ}}}\left(\rho_{e} e+\rho_{f} f+\rho_{h} h, z\right)$. Since

$$
\begin{aligned}
Y_{V_{L^{\circ}}}\left(a(-1) b, z_{2}\right)= & \operatorname{Res}_{z_{0}}\left\{z _ { 0 } ^ { - 1 } \operatorname { R e s } _ { z _ { 1 } } \left\{z_{0}^{-1} \delta\left(\frac{z_{1}-z_{2}}{z_{0}}\right) Y_{V_{L^{\circ}}}\left(a, z_{1}\right) Y_{V_{L^{\circ}}}\left(b, z_{2}\right)\right.\right. \\
& \left.\left.\quad-z_{0}^{-1} \delta\left(\frac{z_{2}-z_{1}}{-z_{0}}\right) Y_{V_{L^{\circ}}}\left(b, z_{2}\right) Y_{V_{L^{\circ}}}\left(a, z_{1}\right)\right\}\right\} \\
= & \operatorname{Res}_{z_{0}} z_{0}^{-1} \operatorname{Res}_{z_{1}} z_{2}^{-1} \delta\left(\frac{z_{1}-z_{0}}{z_{2}}\right) \lambda_{\mathfrak{e}} Y_{V_{L^{\circ}}}\left(b, z_{2}\right) \\
= & \lambda_{\mathfrak{e}} Y_{V_{L^{\circ}}}\left(b, z_{2}\right)
\end{aligned}
$$

this implies that

$$
Y_{V_{L^{\circ}}}\left(a(-1) b, z_{2}\right)=Y_{V_{L^{\circ}}}(a \cdot b, z)
$$

By (23), (24), (25), we can conclude that $V_{L^{\circ}}$ is a $V_{B}$-module. 
Observe that

$$
\begin{aligned}
Y_{V_{L^{\circ}}}\left(e(-1) e, z_{2}\right)= & \operatorname{Res}_{z_{0}}\left\{z _ { 0 } ^ { - 1 } \operatorname { R e s } _ { z _ { 1 } } \left\{z_{0}^{-1} \delta\left(\frac{z_{1}-z_{2}}{z_{0}}\right) Y_{V_{L^{\circ}}}\left(e, z_{1}\right) Y_{V_{L^{\circ}}}\left(e, z_{2}\right)\right.\right. \\
& \left.\left.\quad-z_{0}^{-1} \delta\left(\frac{z_{2}-z_{1}}{-z_{0}}\right) Y_{V_{L^{\circ}}}\left(e, z_{2}\right) Y_{V_{L^{\circ}}}\left(e, z_{1}\right)\right\}\right\} \\
= & \operatorname{Res}_{z_{0}}\left\{z _ { 0 } ^ { - 1 } \operatorname { R e s } _ { z _ { 1 } } \left\{z_{0}^{-1} \delta\left(\frac{z_{1}-z_{2}}{z_{0}}\right) Y\left(e^{\alpha}, z_{1}\right) Y\left(e^{\alpha}, z_{2}\right)\right.\right. \\
& \left.\left.\quad-z_{0}^{-1} \delta\left(\frac{z_{2}-z_{1}}{-z_{0}}\right) Y\left(e^{\alpha}, z_{2}\right) Y\left(e^{\alpha}, z_{1}\right)\right\}\right\} \\
= & Y\left(e_{-1}^{\alpha} e^{\alpha}, z_{2}\right) \\
= & 0 .
\end{aligned}
$$

Hence $V_{L^{\circ}}$ is a $\overline{V_{B}}$-module. This completes the proof of this Lemma.

Lemma 45. Let $W$ be an irreducible $\mathbb{N}$-graded $\overline{V_{B}}$-module. Then $W$ is either isomorphic to $V_{L}$ or $V_{L+\frac{1}{2} \alpha}$.

Proof. Let $W=\oplus_{n=0}^{\infty} W_{(n)}$ be an irreducible $\mathbb{N}$-graded $\overline{V_{B}}$-module. By Lemma 42, $W_{(0)}$ is an irreducible $s l_{2}$-module and the dimension of $W_{(0)}$ is either 1 or 2 . Since $W$ is a $L(1,0)$-module, we can conclude that $W=\oplus_{i=1}^{t} U^{i}$ is a direct sum of irreducible $L(1,0)$-modules $U^{i}$ where $U^{i}=\oplus_{n=0}^{\infty} U_{(n)}^{i}$ is either isomorphic to $V_{L}$ or $V_{L+\frac{1}{2} \alpha}$. Here, $U_{(n)}^{i}=W_{(n)} \cap U^{i}$.

If the dimension of $W_{(0)}$ is 1 then $W$ is isomorphic to $V_{L}$. Now, we assume that the dimension of $W_{(0)}$ is 2 . Then $W$ is either isomorhic to $V_{L} \oplus V_{L}$ or $V_{L+\frac{1}{2} \alpha}$. If $W$ is isomorphic to $V_{L} \oplus V_{L}$ then $W_{(0)}$ is isomorphic to a sum of two trivial $s l_{2}$-modules which is impossible. Hence, $W$ is isomorphic to $V_{L+\frac{1}{2} \alpha}$.

This completes the proof of statement (iii) of Theorem 2 .

\section{Appendices}

\subsection{Vertex operator algebra associated with a rank one even lattice $\mathbb{Z} \alpha$ such that} $(\alpha, \alpha)=2$.

Let $L=\mathbb{Z} \alpha$ be a positive definite even lattice of rank one, i.e., a free abelian group equipped with a $\mathbb{Q}$-valued $\mathbb{Z}$-bilinear form $(\cdot, \cdot)$ such that $(\alpha, \alpha)=2$. We set

$$
\mathbb{H}=\mathbb{C} \otimes_{\mathbb{Z}} L
$$

and extend $(\cdot, \cdot)$ to a $\mathbb{C}$-bilinear form on $\mathbb{H}$. Let

$$
\hat{\mathbb{H}}=\mathbb{H} \otimes \mathbb{C}\left[t, t^{-1}\right] \oplus \mathbb{C} K
$$

be the affine Lie algebra associated to the abelian Lie algebra $\mathbb{H}$ so that

$$
[\alpha(m), \alpha(n)]=2 m \delta_{m+n, 0} K \text { and }[K, \hat{\mathbb{H}}]=0
$$


for any $m, n \in \mathbb{Z}$, where $\alpha(m)=\alpha \otimes t^{m}$. Then $\hat{\mathbb{H}} \geq 0=\mathbb{H} \otimes \mathbb{C}[t] \oplus \mathbb{C} K$ is a commutative subalgebra. For any $\lambda \in \mathbb{H}$, we define a one-dimensional $\hat{\mathbb{H}} \geq 0$-module $\mathbb{C} e^{\lambda}$ such that $\alpha(m) \cdot e^{\lambda}=$ $(\lambda, \alpha) \delta_{m, 0} e^{\lambda}$, and $K \cdot e^{\lambda}=e^{\lambda}$ for $m \geq 0$. We denote by

$$
M(1, \lambda)=U(\hat{\mathbb{H}}) \otimes_{U(\hat{\mathbb{H}} \geq 0)} \mathbb{C} e^{\lambda}
$$

the $\hat{\mathbb{H}}$-module induced from $\hat{\mathbb{H}}^{\geq 0}$-module $\mathbb{C} e^{\lambda}$. We set $M(1)=M(1,0)$. Then there exists a linear map $Y: M(1) \rightarrow$ End $M(1)\left[\left[z, z^{-1}\right]\right]$ such that $(M(1), Y, \mathbf{1}, \omega)$ carries a simple vertex operator algebra structure and $M(1, \lambda)$ becomes an irreducible $M(1)$-module for any $\lambda \in \mathbb{H}$ ([FrLMe1] $)$. The vacuum vector and the Virasoro element are given by $\mathbf{1}=e^{0}$ and $\omega=$ $\frac{1}{4} \alpha(-1)^{2} \mathbf{1}$, respectively.

Let $\mathbb{C}[L]$ be the group algebra of $L$ with a basis $e^{\beta}$ for $\beta \in L$ and multiplication $e^{\beta} e^{\gamma}=e^{\beta+\gamma}$ $(\beta, \gamma \in L)$. The lattice vertex operator algebra associated to $L$ is given by

$$
V_{L}=M(1) \otimes \mathbb{C}[L] .
$$

The dual lattice $L^{\circ}$ of $L$ is the set $\{\lambda \in \mathbb{H} \mid(\alpha, \lambda) \in \mathbb{Z}\}=\frac{1}{2} L$. Note that $L^{\circ}=L \cup\left(L+\frac{1}{2} \alpha\right)$ is the coset decomposition of $L^{\circ}$ with respect to $L$. Also, we set $\mathbb{C}\left[L+\frac{1}{2} \alpha\right]=\oplus_{\beta \in L} \mathbb{C} e^{\beta+\frac{1}{2} \alpha}$. Then $\mathbb{C}\left[L+\frac{1}{2} \alpha\right]$ is a $L$-submodule of $\mathbb{C}\left[L^{\circ}\right]$. We set $V_{L+\frac{1}{2} \alpha}=M(1) \otimes \mathbb{C}\left[L+\frac{1}{2} \alpha\right]$. It was shown in [Bo1, [Do], FrLMe1, $\mathrm{Gu}$ ] that $V_{L}$ is a rational vertex operator algebra. Furthermore, $V_{L}$ and $V_{L+\frac{1}{2} \alpha}$ are the only irreducible modules for $V_{L}$ under the following action: for $\beta \in \mathbb{H}$ write $\beta(z):=\sum_{n \in \mathbb{Z}} \beta(n) z^{-n-1}, z^{\beta}: e^{\gamma} \mapsto z^{(\beta, \gamma)} e^{\gamma}$ and set

$$
Y\left(e^{\beta}, z\right):=\exp \left(\sum_{m=1}^{\infty} \beta(-m) \frac{z^{m}}{m}\right) \exp \left(-\sum_{m=1}^{\infty} \beta(m) \frac{z^{-m}}{m}\right) e^{\beta} z^{\beta},
$$

and for $v=\alpha_{1}\left(-n_{1}\right) \ldots \alpha_{t}\left(-n_{t}\right) \otimes e^{\beta} \in V_{L}\left(n_{i} \geq 1\right)$ set

$$
Y(v, z):=\left(\frac{1}{\left(n_{1}-1\right) !}\left(\frac{d}{d z}\right)^{n_{1}-1} \alpha_{1}(z)\right) \ldots\left(\frac{1}{\left(n_{t}-1\right) !}\left(\frac{d}{d z}\right)^{n_{t}-1} \alpha_{t}(z)\right) Y\left(e^{\alpha}, z\right):
$$

with the usual normal ordering conventions.

\subsection{Vertex operator algebras associated with highest weight representations of affine Lie algebras.}

Let $\mathfrak{g}$ be a simple Lie algebra over $\mathbb{C}, \mathfrak{h}$ its Cartan subalgebra and $\Delta$ the corresponding root system. We fix a set of positive root $\Delta_{+}$and a nondegnerate symmetric invariant bilinear form $\langle\cdot, \cdot\rangle$ of $\mathfrak{g}$ such that the square length of a long root is 2 . Let

$$
\hat{\mathfrak{g}}=\mathfrak{g} \otimes \mathbb{C}\left[t, t^{-1}\right] \oplus \mathbb{C} c
$$

be the affine Lie algebra with Lie bracket defined by

$$
\left[u \otimes t^{m}, v \otimes^{n}\right]=[u, v] \otimes t^{m+n}+m\langle u, v\rangle \delta_{m+n, 0} c .
$$

Here, $u, v \in \mathfrak{g}$ and $m, n \in \mathbb{Z}$ and $c$ is a central element. We will write $u(n)$ for $u \otimes t^{n}$. 
Let $l$ be a complex number such that $l \neq-\check{h}$ where $\check{h}$ is the dual Coxeter number of $\mathfrak{g}$. Let $\mathbb{C}_{l}$ be the one-dimensional $(\mathfrak{g} \otimes \mathbb{C}[t]+\mathbb{C} c)$-module on which $c$ acts as scalar $l$ and $\mathfrak{g} \otimes \mathbb{C}[t]$ acts a zero. Form the generalized Verma $\hat{\mathfrak{g}}$-module

$$
\left.M_{\mathfrak{g}}(l, 0)=U(\hat{\mathfrak{g}}) \otimes_{U(\mathfrak{g} \otimes \mathbb{C}[t]+\mathbb{C} c}\right) \mathbb{C}_{l} .
$$

We define

$$
\begin{aligned}
& Y(u(-1) \otimes 1, z)=\sum_{n \in \mathbb{Z}} u(n) z^{-n-1}, \\
& Y(u(-m-1) \otimes 1, z)=\frac{1}{m !} \frac{d^{m}}{d z^{m}} Y(u(-1) \otimes 1, z) .
\end{aligned}
$$

In general, if $Y(v, z)$ has been defined, we defined $Y(u(-n) v, z)$ for $u \in \mathfrak{g}$ and $n>0$ as

$$
Y(u(-n) v, z)=\operatorname{Res}_{z_{1}}\left\{\left(z_{1}-z_{2}\right)^{-n} Y\left(u, z_{1}\right) Y\left(v, z_{2}\right)-\left(-z_{2}+z_{1}\right)^{-n} Y\left(v, z_{2}\right) Y\left(u, z_{1}\right)\right\} .
$$

We then get a linear map $Y$ from $M_{\mathfrak{g}}(l, 0)$ to $\left(\operatorname{End} M_{\mathfrak{g}}(l, 0)\left[\left[z, z^{-1}\right]\right]\right.$. Set $\mathbf{1}=1 \otimes 1$ and $\omega=\frac{1}{2(l+h)} \sum_{i} v_{i}(-1)^{2} \otimes 1$ where $\left\{v_{i}\right\}$ is an orthonormal basis of $\mathfrak{g}$ with respect to $\langle$,$\rangle .$

\section{Proposition 46. FS, FZ, LLi, Li, MeP]}

(i) $\left(M_{\mathfrak{g}}(l, 0), Y, \mathbf{1}, \omega\right)$ is a vertex operator algebra. The category of weak $M_{\mathfrak{g}}(l, 0)$-modules in the sense that all axioms defining the notion of module except those involving grading hold is canonically equivalent to the category of restricted $\hat{\mathfrak{g}}$-modules of level $l$ in the sense that for every vector $w$ of the module, $\left(\mathfrak{g} \otimes t^{n} \mathbb{C}[t]\right) w=0$ for $n$ sufficiently large.

(ii) Let $J_{l, 0}$ be the maximal proper submodule of $M_{\mathfrak{g}}(l, 0)$. Let $e_{\theta}$ be an element in the root space $\mathfrak{g}_{\theta}$ of the maximal root $\theta$. The space $J_{l, 0}$ is generated by the vector $e_{\theta}(-1)^{l+1} \mathbf{1}$, i.e., every element in $J_{l, 0}$ can be written as a linear combination of elements of type

$$
u_{1}\left(-n_{1}\right) \ldots . u_{m}\left(-n_{t}\right) e_{\theta}(-1)^{l+1} \mathbf{1} .
$$

Note that 1 and $\omega$ are not members of $J_{l, 0}$.

Given a $\mathfrak{g}$-module $W$, we will write $u_{W}(n)$ for the operator on $W$ corresponding to $u \otimes t^{n}$ for $u \in \mathfrak{g}$ and $n \in \mathbb{Z}$ and we set $u_{W}(z)=\sum_{n \in \mathbb{Z}} u_{W}(n) z^{-n-1} \in(\operatorname{End} W)\left[\left[z, z^{-1}\right]\right]$. $W$ is a restricted module of level $l$ if $c$ acts as $l$ and for every $u \in \mathfrak{g}, w \in W, u(n) w=0$ for $n$ sufficiently large.

We set $L(l, 0)=M_{\mathfrak{g}}(l, 0) / J_{l, 0}$.

\section{Proposition 47. [FZ, LLi, DoL}

(i) $L(l, 0)$ is a rational vertex operator algebra.

(ii) Let $\alpha \in \Delta$ be a long root, i.e., $\langle\alpha, \alpha\rangle=2$ and let $e \in \mathfrak{g}_{\alpha}$. Then $e(-1)^{l+1} \mathbf{1} \in J_{l, 0}$. In particular, $e_{\theta}(-1)^{l+1} \mathbf{1}, f_{\theta}(-1)^{l+1} \mathbf{1} \in J_{l, 0}$. Moreover, $Y(e, x)^{l+1}=0$ on $L(l, 0)$. For any module $W$ for $L(l, 0)$ viewed as a vertex algebra, $Y_{W}(e, z)^{l+1}=0$. In particular, $Y_{W}\left(e_{\theta}, z\right)^{l+1}=$ $Y_{W}\left(f_{\theta}, z\right)^{l+1}=0$.

(iii) If $\mathfrak{g}=s l_{2}$ and $l=1$, then $V_{L}$ and $V_{L+\frac{1}{2} \alpha}$ are the only irreducible $L(1,0)$-modules. 


\section{REFERENCES}

[A1] Abe, T., A $\mathbb{Z}_{2}$-orbifold model of the symplectic fermionic vertex operator superalgebra, Math. Z. 255 (2007), 755-792.

[A2] Abe, T., $C_{2}$-cofiniteness of 2-cyclic permutation orbifold models, Commun. Math. Phys. 317 (2013), no. $2,425-445$.

[ABD] Abe, T., Buhl, G., and Dong, C., Rationality, regularity and $C_{2}$-cofiniteness, Trans. Amer. Math. Soc. 356 (2004) 3391-3402.

[AdM1] Adamovic, D., and Milas, A., On the triplet vertex algebra $W(p)$, Advances in Math. 217 (2008), 2664-2699.

[AdM2] Adamovic, D., and Milas, A., The $N=1$ triplet vertex operator superalgebras, Comm. Math. Phys. 288 (2009) 225-270.

[AdM3] Adamovic, D., and Milas, A., On $W$-Algebras Associated to $(2, p)$ Minimal Models and Their Representations, IMRN (2010) $20: 3896-3934$.

[AdM4] Adamovic, D., and Milas, A., On $W$-algebra extensions of $(2, p)$ minimal models: $p>3$, J. Alg. 344 (2011), 313-332.

[Ba] Barnes, D., On Levi's Theorem for Leibniz algebras, Bull. Aust. Math. Soc., 86(2) (2012), $184-185$.

[Bo1] Borcherds, R. E., Vertex algebras, Kac-Moody algebras, and the Monster, Proc. Natl. Acad. Sci. USA 83 (1986), 3068-3071.

[Bo2] Borcherds, R. E. Monstrous moonshine and monstrous Lie algebras, Invent. Math. 109 (1992), 405444 .

[Br1] Bressler, P., Vertex algebroids I, arXiv: math.AG/0202185.

[Br2] Bressler, P., Vertex algebroids II, arXiv: math.AG/0304115.

[Bu] Buhl, G., A spanning set for VOA modules, J. Algebra 254 (2002) no. 1, 125-151.

[CF] Carqueville, N. and Flohr, M., Nonmeromorphic operator product expansion and $C_{2}$ - cofiniteness for a family of W-algebras, J. Phys. A: Mathematical and General 39 (2006): 951.

[DMS] Demir, I., Misra, K.C., and Stitzinger, E. On Some Structure of Leibniz Algebras, Contemporary Math. 623 (2014), 41-54.

[Do] Dong, C., Vertex algebras associated with even lattices, J. Algebra 160 (1993), 245-265.

[DoL] Dong, C., and Lepowsky, J., Generalized Vertex Algebras and Relative Vertex Operators, Progress in Math. Vol. 112, Birkhäuser, Boston, 1993.

[DoLiM1] Dong, C., Li, H.-S., and Mason, G., Modular-Invariance of Trace Functions in Orbifold Theory and Generalized Moonshine, Commun. Math. Phys. 214, 1-56 (2000).

[DoLiM2] Dong, C., Li, H.-S., and Mason, G., Vertex Lie algebra, vertex Poisson algebras and vertex algebras, in: Recent Developments in Infinite-Dimensional Lie Algebras and Conformal Field Theory, Proceedings of an International Conference at University of Virginia, May 2000, Contemporary Math. 297 (2002), 69-96.

[DoM1] Dong, C., and Mason, G., On Quantum Galois Theory, Duke. Math. J., 86 (1997), 305-321.

[DoM2] Dong, C., and Mason, G., Local and Semilocal Vertex Operator Algebras, J. Alg., 280 (2004), 350366.

[FM] Fialowski, A., and Mihalka, E. Z. Representations of Leibniz Algebras, Algebras and Representation Theory 18 (2015), 477-490.

[FFHST] Fjelstad, J., Fuchs, J., Hwang, S., Semikhatov, A.M., and Yu Tipunin, I., Logarithmic conformal field theories via logarithmic deformations, Nuclear Phys. B 633 (2002), 379-413.

[FGST1] Feigin, B.L., Gaĭnutdinov, A.M. Semikhatov, A.M.and Yu Tipunin, I., The Kazhdan-Lusztig correspondence for the representation category of the triplet W-algebra in logorithmic conformal field theories. (Russian) Teoret. Mat. Fiz. 148 (2006), no. 3, 398-427.

[FGST2] Feigin, B.L., Gaĭnutdinov, A.M. Semikhatov, A.M.and Yu Tipunin, I. , Logarithmic extensions of minimal models: characters and modular transformations. Nuclear Phys. B 757 (2006), 303-343.

[FGST3] Feigin, B.L., Gaínutdinov, A.M. Semikhatov, A.M.and Yu Tipunin, I., Modular group representations and fusion in logarithmic conformal field theories and in the quantum groupcenter. Comm. Math. Phys. 265 (2006), 47-93. 
[FS] Fuchs, J., and Schweigert, C., The action of outer automorphisms on bundles of chiral blocks, Commun. Math. Phys. 206 (1999), 691-736.

[FKRW] Frenkel, E., Kac, V., Ratiu, A., and Wang, W., $W_{1+\infty}$ and $W_{\left(g l_{\infty}\right)}$ with central charge $N$, Comm. Math. Phys. 170 (1995) 337-357.

[FrLMe1] Frenkel, I., Lepowsky, J., and Meurman, A., A natural representation of the Fisher-Griess Monster with the modular function $J$ as character, Proc. Acad. Sci. USA 81 (1984), 3256-3260.

[FrLMe1] Frenkel, I., Lepowsky, J., and Meurman, A., Vertex Operator Algebras and the Monster, Pure and Applied Math., Vol.134, Academic Press (1988).

[FZ] Frenkel, I., and Zhu, Y.-C., Vertex operator algebras associated to representations of affine and Virasoro algebra, Duke Math. J. 66 (1992), 123-168.

[GN] Gaberdiel and A. Neitzke, Rationality; quasi rationality, and finite $W$-algebras, Commun. Math. Phys. B168, 407-436.

[GMS] Gorbounov, V., Malikov, F., and Schechtman, V., Gerbes of chiral differential operators, II, Vertex algebroids, Invent. Math. 155 (2004), 605-680.

[Gu] H. Guo, On Abelian Intertwining Algebras and Modules, Ph. D. Dissertation, Rutgers University, 1994.

[JY] Jitjankarn, P., and Yamskulna, G., On Indecomposable Non-Simple $\mathbb{N}$-graded Vertex Algebra, arXiv: 1907.11627.

[K] Kac, V., Infinite dimensional Lie algebras, Cambridge University Press, 1990.

[LLi] Lepowsky, L., and Li, H.-S., Introduction to Vertex Operator Algebras and Their Representations, Progr. Math., vol. 227, Birkhäuser, Boston (2003).

[Li] Li, H.-S., Local systems of vertex operators, vertex superalgebras and modules, J. Pure Appl. Alg. 109 (1996), 143-195.

[LiY1] Li, H.-S., and Yamskulna, G., On certain vertex algebras and their modules associated with vertex algebroids, J. Alg. 283 (2005) 367-398.

[LiY2] Li, H.-S., and Yamskulna, G.,Twisted modules of vertex algebras associated with vertex algebroids, Pac. J. Math., 220, No. 1, 1999-222 (2007).

[MS1] Malikov, F., and Schechtman, V., Chiral de Rham complex, II. Differential Topology, Infinite Dimensional Lie Algebras, and Applications, Amer. Math. Soc. Transl. Ser 2, vol 194, Amer. Math. Soc., Providence, RI, 1999, 149-188.

[MS2] Malikov, F., and Schechtman, V., Chiral Poincare duality, Math. Res. Lett. 6 (1999), 533-546.

[MSV] Malikov, F., Schecthman, V., and Vaintrob, A., Chiral de Rham complex, Comm. Math. Phys. 204 (1999)439-473.

[MeP] Meurman, A., and Primc, M., Annihilating Fields of Standard Modules of $\tilde{s l}(2, \mathbb{C})$ and Combinatorial Indentities, Mem. Amer. Math. Soc., vol 652, 1999.

[Mi1] Miyamoto, M., Modular invariance of vertex operator algebras satisfying $C_{2}$-cofiniteness, Duke Math. J. 122 (2004), no. 1, 51-91.

[Mi2] Miyamoto, M., A $C_{2}$-cofinite of cyclic orbifold models. Comm. Math. Phys. 335 no. 3 (2015), 12791286.

[Mi3] Miyamoto, M., $C_{2}$-cofiniteness of orbifold models for finite groups, arXiv: math.QA/1812.00570.

[Z] Zhu, Y.-C., Modular invariance of characters of vertex operator algebras, J. Amer. Math. Soc. 9 (1996), 237-302.

School of Science, Walailak University, Nakhon Si Thammarat, Thailand

E-mail address: jitjankarn@gmail.com

Department of Mathematics, Illinois State University, Normal, IL, USA

E-mail address: gyamsku@ilstu.edu 